\title{
THE STRUCTURE OF QUASI-MULTIPLIERS OF $C^{*}$-ALGEBRAS
}

\author{
HUAXIN LIN
}

\begin{abstract}
Let $A$ be a $C^{*}$-algebra and $A^{* *}$ its enveloping $W^{*}$-algebra. Let $\operatorname{LM}(A)$ be the left multipliers of $A, \operatorname{RM}(A)$ the right multipliers of $A$ and $\mathrm{QM}(A)$ the quasi-multipliers of $A$. A question was raised by Akemann and Pedersen [1] whether $\mathrm{QM}(A)=\mathrm{LM}(A)+\mathrm{RM}(A)$. McKennon [20] gave a nonseparable counterexample. L. Brown [6] shows the answer is negative for stable (separable) $C^{*}$-algebras also.

In this paper, we mainly consider $\sigma$-unitial $C^{*}$-algebras. We give a criterion for $\mathrm{QM}(A)=\mathrm{LM}(A)+\mathrm{RM}(A)$. In the case that $A$ is stable, we give a necessary and sufficient condition for $\mathrm{QM}(A)=\mathrm{LM}(A)+\mathrm{RM}(A)$. We also give answers for other $C^{*}$-algebras.
\end{abstract}

\section{INTRODUCTION AND PRELIMINARIES}

Definition 1.1. Let $A$ be a $C^{*}$-algebra and $A^{* *}$ its enveloping von Neumann algebra. An element $x$ in $A^{* *}$ is called a multiplier of $A$ if $x a \in A$ and $a x \in A$ for all $a \in A$. Similarly, $x$ is a left multiplier if $x a \in A$, for all $a \in A, x$ is a right multiplier if $a x \in A$, for all $a \in A$, and $x$ is a quasimultiplier if $a x b \in A$, for all $a, b \in A$. We denote the sets of multipliers, left multipliers, right multipliers and quasi-multipliers by $\mathbf{M}(A), \operatorname{LM}(A), \operatorname{RM}(A)$ and $\mathrm{QM}(A)$, respectively.

If $\pi: A \rightarrow B(H)$ is a faithful representation, then the extension of $\pi$ to $A^{* *}$ maps $\mathrm{M}(A), \mathrm{LM}(A), \operatorname{RM}(A)$ and $\mathrm{QM}(A)$ isometrically onto the sets of operators in $B(H)$ that satisfy the appropriate multiplication properties relative to $\pi(A)$. Each set $\mathrm{M}(A), \operatorname{LM}(A), \operatorname{RM}(A)$ and $\mathrm{QM}(A)$ is equipped with a natural weak topology.

Definition 1.2. Let $A$ be a $C^{*}$-algebra and $A^{* *}$ its enveloping von Neumann algebra. The strict topology on $A^{* *}$ is generated by the seminorms $x \rightarrow\|x a\|$ and $x \rightarrow\|a x\|, a \in A$. Similarly, we have the left strict topology, generated by the seminorms $\|x a\|$, the right strict topology, generated by $\|a x\|$, and the quasi-strict topology, generated by $\|a x b\|, a, b \in A$.

Received by the editors June 27, 1986 and, in revised form, March 10, 1988.

1980 Mathematics Subject Classification (1985 Revision). Primary 46L05.

This paper is the major part of the author's thesis [19] under the supervision of Professor Lawrence G. Brown. 
$\mathrm{M}(A)$ is the strict closure of $A, \mathbf{L M}(A)$ is the left strict closure of $A$, $\operatorname{RM}(A)$ is the right closure of $A$ and $\mathrm{QM}(A)$ is the quasi-strict closure of $A$. For detailed expositions of these results the reader is referred to $[1,2,8$ and 21].

$\mathrm{LM}(A), \mathrm{RM}(A)$ and $\mathrm{QM}(A)$ are norm closed subspaces in $A^{* *} ; \mathrm{QM}(A)$ is *-invariant, whereas $(\mathrm{LM}(A))^{*}=\mathrm{RM}(A)$. Moreover, $\mathrm{LM}(A)$ and $\operatorname{RM}(A)$ are Banach algebras. The best behaved class is $\mathrm{M}(A)$ which is a $C^{*}$-algebra. It is clear that $\mathrm{M}(A)=\mathrm{LM}(A) \cap \mathrm{RM}(A)$ and that $\mathrm{LM}(A)+\mathrm{RM}(A) \subset \mathrm{QM}(A)$. The question was raised by Akemann and Pedersen [1] in 1973 whether $\mathrm{QM}(A)=$ $\mathrm{LM}(A)+\mathrm{RM}(A)$. McKennon [20] gave a nonseparable counterexample in 1978. Recently, L. Brown. showed [6] that even when $A$ is stable and separable, $\mathrm{QM}(A)$ may not equal $\mathrm{LM}(A)+\mathrm{RM}(A)$.

In this paper, we give exact conditions for $\mathrm{QM}(A)=\mathrm{LM}(A)+\mathrm{RM}(A)$ and for $\mathrm{QM}(A) \neq \mathrm{LM}(A)+\mathrm{RM}(A)$.

Definition 1.3. A topological space $X$ is scattered if every closed subset of $X$ has a relatively isolated point.

Definition 1.4. Let $X$ be a scattered topological space. We define $X_{[0]}=X$, $X_{[1]}=X \backslash\{$ isolated points of $X\}$. If $X_{[\alpha]}$ is defined for some ordinal number $\alpha$, define $X_{[\alpha+1]}=X_{[\alpha]} \backslash\left\{\right.$ isolated points of $\left.X_{[\alpha]}\right\}$, if $\beta$ is a limit ordinal, define $X_{[\beta]}=\bigcap_{\alpha<\beta} X_{[\alpha]}$.

Definition 1.5. Let $X$ be a scattered topological space. We define $\lambda(X)=\alpha$, if $\alpha$ is the least ordinal such that $X_{[\alpha]}$ is discrete. Since $X$ is scattered, $\lambda(X)$ is well defined.

Definition 1.6. Let $Y_{1}=\{0,1 / n, n=1,2, \ldots\}$, a subset of $[0,1]$ with the usual topology, and let $Y_{2}$ be the one-point compactification of the disjoint union of countably many copies of $Y_{1}$. If $Y_{\alpha}$ is defined for some ordinal number $\alpha$, define $Y_{\alpha+1}$ as the one-point compactification of the disjoint union of countably many copies of $Y_{\alpha}$. If $\beta$ is a limit ordinal, define $Y_{\beta}$ as the one-point compactification of the disjoint union of $Y_{\alpha}, \alpha<\beta$. We also define $Z_{\alpha}^{(m)}$ to be the union of $m$ disjoint copies of $Y_{\alpha}$.

Theorem 1.7 [17] (or see [19, Theorem 1.9]). Let $X$ be a countable, compact Huasdorff space with $\lambda(X)=\alpha \geq 1$ and assume that $X_{[\alpha]}$ consists of $n$ points. Then $X$ is homeomorphic to $Z_{\alpha}^{(n)}$.

Let $\{X, A(t), \mathscr{F}\}$ be a continuous field of $C^{*}$-algebras with $X$ a locally compact Hausdorff space. Let $A=C_{0}(X, A(t), \mathscr{F})$ be the set of all continuous cross sections of $\{X, A(t), \mathscr{F}\}$ vanishing at infinity. Then $A$ is a $C^{*}$-algebra.

We say a bounded cross section $x$ in the bundle

$$
\{X, \operatorname{LM}(A(t))\} \quad(\{X, \mathrm{RM}(A(t))\},\{X, \mathrm{QM}(A(t))\})
$$

is left-strictly (right-strictly, quasi-strictly) continuous at $t_{0}$, if for every $a \in \mathscr{F}$, $x a(a x, a x a)$ is continuous at $t_{0}$. We denote by $C^{b}\left(X, \operatorname{LM}(A(t))_{\mathrm{L} . \mathrm{S} .}, \mathscr{F}\right)$ 
$\left(C^{b}\left(X, \mathrm{RM}(A(t))_{\mathrm{R} . S .}, \mathscr{F}\right), C^{b}\left(X, \mathrm{QM}(A(t))_{\mathrm{Q} . \mathrm{S} .}, \mathscr{F}\right)\right)$ the set of all bounded left-strictly (right-strictly, quasi-strictly) continuous cross sections in

$$
\{X, \mathbf{L M}(A(t))\} \quad(\{X, \mathbf{R M}(A(t))\},\{X, \mathrm{QM}(A(t))\}) .
$$

Let $A=C_{0}(X, A(t), \mathscr{F})$. Exactly as in [2,3.3] we obtain

Theorem 1.8 (see $[19, \S 1.3]$ also).

$$
\begin{aligned}
& C^{b}\left(X, \mathrm{LM}(A(t))_{\text {L.S. }}, \mathscr{F}\right)=\mathrm{LM}(A) ; \\
& C^{b}\left(X, \operatorname{RM}(A(t))_{\mathrm{R} . S .}, \mathscr{F}\right)=\mathrm{RM}(A) ; \\
& C^{b}\left(X, \mathrm{QM}(A(t))_{\mathrm{Q} . S .}, \mathscr{F}\right)=\mathrm{QM}(A) .
\end{aligned}
$$

\section{A CRITERION FOR $\mathrm{QM}(A)=\mathrm{LM}(A)+\mathrm{RM}(A)$}

Let $A$ be a $\sigma$-unital $C^{*}$-algebra, and $a$ a strictly positive element, $0<$ $a \leq 1$. For each $n$ let $f_{n}$ be a continuous function such that $f_{n}(t)=1$ if $t \geq 1 / n, f_{n}(t)=0$ if $0 \leq t \leq 1 /(n+1)$ and $f_{n}$ is linear in $[1 /(n+1), 1 / n]$. Define $e_{n}=f_{n}(a)$. Then $\left\{e_{n}\right\}$ is an approximate identity for $A$. Moreover $e_{m} e_{n}=e_{n} e_{m}=e_{n}$, if $m>n$.

Lemma 2.1. Let $A$ be a $\sigma$-unital $C^{*}$-algebra and $\left\{e_{n}\right\}$ an approximate identity for $A$ satisfying $e_{m} e_{n}=e_{n} e_{m}=e_{n}$, if $m>n$. Suppose that $y \in \mathrm{QM}(A)$, then $y \in \operatorname{LM}(A)$ if and only if there exists an increasing sequence $\left\{n_{k}\right\}$ of nonnegative integers such that

$$
\sum_{k=1}^{\infty}\left(1-e_{n_{k+1}}\right) y\left(e_{n_{k}}-e_{n_{k-1}}\right)
$$

converges in norm to an element of $A$ where $e_{n_{0}}=0$.

Proof. Assume that $y \in \operatorname{LM}(A)$. For every $m, y e_{m} \in A$. Hence there is $m^{\prime}$ such that $\left\|\left(1-e_{m^{\prime}}\right) y e_{m}\right\|<1 / 2^{m}$. Therefore we can recursively define $n_{1}<n_{2}<\cdots$ so that

$$
\left\|\left(1-e_{n_{k+1}}\right) y e_{n_{k}}\right\|<\frac{1}{2^{k}} .
$$

This implies that $\sum_{k=1}^{\infty}\left(1-e_{n_{k+1}}\right) y\left(e_{n_{k}}-e_{n_{k-1}}\right)$ is norm convergent to an element in $A$.

For the converse, let $z=y-\sum_{k=1}^{\infty}\left(1-e_{n_{k+1}}\right) y\left(e_{n_{k}}-e_{n_{k-1}}\right)$. For fixed $n$, let $m$ be the least integer such that $n_{m}>n$. Then

$$
\begin{aligned}
z e_{n} & =y e_{n}-\sum_{k=1}^{m}\left(1-e_{n_{k+1}}\right) y\left(e_{n_{k}}-e_{n_{k-1}}\right) e_{n} \\
& =\sum_{k=1}^{m}\left[y\left(e_{n_{k}}-e_{n_{k-1}}\right) e_{n}-\left(1-e_{n_{k+1}}\right) y\left(e_{n_{k}}-e_{n_{k-1}}\right) e_{n}\right] \\
& =\sum_{k=1}^{m} e_{n_{k+1}} y\left(e_{n_{k}}-e_{n_{k-1}}\right) e_{n} .
\end{aligned}
$$


Since $y \in \mathrm{QM}(A)$, we conclude that $z e_{n} \in A$, for all $n$. Hence $z \in \mathrm{LM}(A)$. It follows that

$$
y=z+\sum_{k=1}^{\infty}\left(1-e_{n_{k+1}}\right) y\left(e_{n_{k}}-e_{n_{k-1}}\right) \in \operatorname{LM}(A) .
$$

Lemma 2.2. Let $A$ be a $\sigma$-unital $C^{*}$-algebra and $\left\{e_{n}\right\}$ an approximate identity for $A$ satisfying $e_{m} e_{n}=e_{n} e_{m}=e_{n}$, if $m>n$. Suppose that $x_{n} \in \mathrm{QM}(A)$ with $\left\|x_{n}\right\| \leq M$ for some $M, j$ is an integer and $0<\alpha \leq 1$. Then

$$
\sum_{n=1}^{\infty}\left(e_{n+j+1}-e_{n+j}\right)^{\alpha} x_{n}\left(e_{n}-e_{n-1}\right)^{\alpha}
$$

converges strictly.

Proof. Let $P_{s}$ be the range projection of $\left(e_{s}-e_{s-1}\right)$ and

$$
y_{s}=\left(e_{s+j+1}-e_{s+j}\right)^{\alpha} x_{s}\left(e_{s}-e_{s-1}\right)^{\alpha} .
$$

Clearly $P_{s} \cdot P_{s+2+i}=0$ for $i=0,1,2, \ldots$. Suppose that $A \subset B(H)$ and $f \in H$. Then

$$
\begin{aligned}
\left\|\sum_{\substack{s=2 k \\
s \leq N}} y_{s} f\right\|^{2} & =\left\|\sum_{\substack{s=2 k \\
s \leq N}} P_{s+j+1} y_{s} P_{s} f\right\|^{2} \\
& =\sum_{\substack{s=2 k \\
s \leq N}}\left\|P_{s+j+1} y_{s} P_{s} f\right\|^{2} \leq M^{2}\|f\|^{2}
\end{aligned}
$$

for all $N$. Similarly

$$
\left\|\sum_{\substack{s=2 k+1 \\ s \leq N}} y_{s} f\right\|^{2} \leq M^{2}\|f\|^{2} \text { for all } N .
$$

So $\left\{\left\|\sum_{n=1}^{N} y_{n}\right\|\right\}$ is bounded. For fixed $m$, if $N>m+1$, then

$$
e_{m} \sum_{n=N}^{N+k} y_{n}=\sum_{n=N}^{N+k} y_{n} e_{m}=0
$$

for every $k$. Hence $\sum_{n=1}^{\infty} y_{n}$ converges strictly.

Theorem 2.3. Let $A$ be a $\sigma$-unital $C^{*}$-algebra and $\left\{e_{n}\right\}$ and approximate identity for $A$ satisfying $e_{m} e_{n}=e_{n} e_{m}=e_{n}$, if $m>n$. Then $\mathrm{QM}(A)=$ $\mathrm{LM}(A)+\mathrm{RM}(A)$ if and only if for every $x \in \mathrm{QM}(A)_{\text {s.a. }}$, there exists an increasing sequence $\left\{n_{k}\right\}$ of nonnegative integers such that

$$
\sum_{k=1}^{\infty}\left(1-e_{n_{k}}\right) x\left(e_{n_{k}}-e_{n_{k-1}}\right)
$$

converges strictly $\left(e_{n_{0}}=0\right)$. 
Proof. Let $x \in \mathrm{QM}(A)_{\text {s.a. }}$ and $n_{1}<n_{2}<\cdots$ be chosen such that $\sum_{k=1}^{\infty}\left(1-e_{n_{k}}\right) x\left(e_{n_{k}}-e_{n_{k-1}}\right)$ converges strictly. Let $x_{k}=\left(1-e_{n_{k}}\right) x\left(e_{n_{k}}-e_{n_{k-1}}\right)$. Since $\sum_{k=1}^{N} x_{k} \in \operatorname{RM}(A)$ for all $N$, we conclude that $\sum_{k=1}^{\infty} x_{k} \in \mathbf{R M}(A)$. For a fixed $m$, suppose that $k_{0}$ is the least integer such that $n_{k_{0}}>m$. Then

$$
\begin{aligned}
\left(x-\sum_{k=1}^{\infty} x_{k}\right) e_{m} & =x e_{m}-\sum_{k=1}^{k_{0}} x_{k} e_{m} \\
& =\sum_{k=1}^{k_{0}} e_{n_{k}} x\left(e_{n_{k}}-e_{n_{k-1}}\right) e_{m} \in A .
\end{aligned}
$$

Hence $x-\sum_{k=1}^{\infty} x_{k} \in \mathrm{LM}(A)$. This implies that $\mathrm{QM}(A)_{\text {s.a. }} \subset \mathrm{LM}(A)+\mathrm{RM}(A)$, and hence $\mathrm{QM}(A)=\mathrm{LM}(A)+\mathrm{RM}(A)$.

Next assume that $\mathrm{QM}(A)=\mathrm{LM}(A)+\mathrm{RM}(A)$. Equivalently, $\mathrm{QM}(A)_{\text {s.a. }}=$ $\operatorname{Re} \operatorname{LM}(A)$. Let $x \in \mathrm{QM}(A)_{\text {s.a. }}$. Thus there is $y \in \operatorname{LM}(A)$ such that $x=$ $y+y^{*}$. By Lemma 2.1, we can choose $n_{1}<n_{2}<\cdots$ such that the elements $y_{k}=\left(1-e_{n_{k+1}}\right) y\left(e_{n_{k}}-e_{n_{k-1}}\right)$ satisfy $\left\|y_{k}\right\|<2^{-k}$, whence $\sum_{k=1}^{\infty} y_{k} \in A$. By Lemma $2.2 \sum_{k=1}^{\infty}\left(e_{n_{k+1}}-e_{n_{k}}\right) y\left(e_{n_{k}}-e_{n_{k-1}}\right)$ converges strictly. Hence $\sum_{k=1}^{\infty}\left(1-e_{n_{k}}\right) y\left(e_{n_{k}}-e_{n_{k-1}}\right)$ converges strictly.

Let

$$
\begin{gathered}
y_{k j}=\left(e_{n_{k}}-e_{n_{k-1}}\right) e_{n_{j+1}} y\left(e_{n_{j}}-e_{n_{j-1}}\right), \\
y_{k}^{(1)}=\left(e_{n_{k}}-e_{n_{k-1}}\right) e_{n_{k+1}} y\left(1-e_{n_{k}}\right)\left(e_{n_{k}}-e_{n_{k-1}}\right)
\end{gathered}
$$

and

$$
y_{k}^{(2)}=\left(e_{n_{k}}-e_{n_{k-1}}\right) e_{n_{k+2}} y\left(1-e_{n_{k}}\right)\left(e_{n_{k+1}}-e_{n_{k}}\right) .
$$

Then by Lemma 2.2,

$$
\sum_{k=1}^{\infty} y_{k}^{(1)}, \quad \sum_{k=1}^{\infty} y_{k}^{(2)} \text { and } \quad \sum_{k=1}^{\infty} \sum_{j=1}^{k+1} y_{k j}=\sum_{k=1}^{\infty} \sum_{j=k+2}^{k+1} y_{k j}
$$

converge strictly. Since

$$
\sum_{k=1}^{\infty} \sum_{j=k+2}^{\infty} y_{k j}+\sum_{k=1}^{\infty} \sum_{j=1}^{k+1} y_{k j}=\sum_{j=1}^{\infty} e_{n_{j+1}} y\left(e_{n_{j}}-e_{n_{j-1}}\right)=y-\sum_{j=1}^{\infty} y_{j} .
$$

We conclude that $\sum_{k=1}^{\infty} \sum_{j=k+2}^{\infty} y_{k j}$ converges strictly. Thus

$$
\begin{aligned}
\sum_{k=1}^{\infty} & \left(e_{n_{k}}-e_{n_{k-1}}\right)\left(y-\sum_{j=1}^{\infty} y_{j}\right)\left(1-e_{n_{k}}\right) \\
= & \sum_{k=1}^{\infty} \sum_{j=1}^{\infty}\left(e_{n_{k}}-e_{n_{k-1}}\right) e_{n_{j+1}} y\left(e_{n_{j}}-e_{n_{j-1}}\right)\left(1-e_{n_{k}}\right) \\
= & \sum_{k=1}^{\infty} \sum_{j=k+2}^{\infty} y_{k j}+\sum_{k=1}^{\infty} y_{k}^{(1)}=\sum_{k=1}^{\infty} y_{k}^{(2)}
\end{aligned}
$$


converges strictly. So

$$
\sum_{k=1}^{\infty}\left(1-e_{n_{k}}\right)\left(y^{*}-\sum_{j=1}^{\infty} y_{j}^{*}\right)\left(e_{n_{k}}-e_{n_{k-1}}\right)
$$

converges strictly. Since $\left(1-e_{n_{k}}\right)\left(e_{n_{j}}-e_{n_{j-1}}\right)=0$ if $k>j$ and

$$
\left(1-e_{n_{j+1}}\right)\left(e_{n_{k}}-e_{n_{k-1}}\right)=0, \quad \text { if } j \geq k,
$$

we have

$$
\sum_{k=1}^{\infty}\left(1-e_{n_{k}}\right)\left(\sum_{j=1}^{\infty} y_{j}\right) *\left(e_{n_{k}}-e_{n_{k-1}}\right)=0 .
$$

Finally, since $x=y+y^{*}, \sum_{k=1}^{\infty} x_{k}$ converges strictly. This completes the proof.

\section{LIFTING AND HEREDITARY PROPERTIES}

Considering the problem $\mathrm{QM}(A)=\mathrm{LM}(A)+\mathrm{RM}(A)$, one may ask the following questions:

(i) If $I$ is an ideal of $A$ such that $\mathrm{QM}(A / I)=\mathrm{LM}(A / I)+\mathrm{RM}(A / I)$ and $\mathrm{QM}(I)=\mathrm{LM}(I)+\mathrm{RM}(I)$, does it follow that $\mathrm{QM}(A)=\mathrm{RM}(A)+\mathrm{LM}(A)$ ?

(ii) If $\mathrm{QM}(A)=\mathrm{LM}(A)+\mathrm{RM}(A)$, does it follow tht $\mathrm{QM}(B)=\mathrm{LM}(B)+$ $\operatorname{RM}(B)$ for $B$ in $A$ ?

In this section, we shall show that (i) has a positive answer under a suitable assumption on $A$, and for some special $B$ 's, (ii) also has a positive answer. However, in general (ii) has a negative answer, as we shall see in Example 8.2.

Theorem 3.1. Let $A$ be a $\sigma$-unital $C^{*}$-algebra and $B$ a $C^{*}$-subalgebra of $A$ such that the hereditary $C^{*}$-subalgebra generated by $B$ is $A$ itself. If $\mathrm{QM}(A)=$ $\mathbf{L M}(A)+\mathbf{R M}(A)$, then $\mathrm{QM}(B)=\mathbf{L M}(B)+\mathbf{R M}(B)$.

Proof. Let $\tilde{A}$ and $\widetilde{B}$ be $C^{*}$-algebras obtained by adding identities to $A$ and $B$. Since the hereditary $C^{*}$-subalgebra generated by $B$ is $A$ itself, $B$ contains a strictly positive element of $A$, say $a$. It follows that $A$ and $B$ share a common approximate identity $\left\{e_{n}\right\}$ satisfying $e_{n} e_{m}=e_{m} e_{n}=e_{n}$, if $m>n$. Since $e_{n}$ converges weakly to the identity of $A$ and the identity of $B$ in $A^{* *}$, $\widetilde{A}$ and $\widetilde{B}$ have the same identity. Thus

$$
\mathrm{QM}(B)_{\text {s.a. }}=\left[\left(\widetilde{B}_{\text {s.a. }}\right)^{m}\right]^{-} \cap\left[\left(\widetilde{B}_{\text {s.a. }}\right)_{m}\right]^{-} \subset\left[\left(\widetilde{A}_{\text {s.a. }}\right)^{m}\right]^{-} \cap\left[\left(\widetilde{A}_{\text {s.a. }}\right)_{m}\right]^{-}=\mathrm{QM}(A)_{\text {s.a. }}
$$

(see [1, Theorem 4.1]). Since $A$ and $B$ have the same approximate identity $\left\{e_{n}\right\}$, we can apply Theorem 2.3 to obtain the desired conclusion.

Let $A$ be a $C^{*}$-algebra and $I$ a closed ideal. We shall denote $\mathrm{M}(A) \cap I^{* *}$, $\operatorname{LM}(A) \cap I^{* *}, \operatorname{RM}(A) \cap I^{* *}$ and $\mathrm{QM}(A) \cap I^{* *}$ by $\mathrm{M}(A, I), \operatorname{LM}(A, I), \operatorname{RM}(A, I)$ and $\mathrm{QM}(A, I)$, respectively. If $x \in \mathrm{M}(A, I)$, and $a \in A$, one can see that $a x$, $x a \in I$. Moreover, if $x \in \operatorname{LM}(A, I), x a \in I$, etc. 
Lemma 3.2. Let $A$ be a $\sigma$-unital $C^{*}$-algebra and $I$ an ideal of $A$. Then $\mathrm{QM}(A, I)=\mathrm{LM}(A, I)+\operatorname{RM}(A, I)$ if one of the following holds:

(i) $\mathrm{QM}(A)=\mathrm{LM}(A)+\mathrm{RM}(A)$ or

(ii) $\mathrm{QM}(I)=\mathrm{LM}(I)+\mathrm{RM}(I)$.

Proof. Let $\left\{e_{n}\right\}$ be an approximate identity of $A$ satisfying $e_{m} e_{n}=e_{n} e_{m}=e_{n}$, if $m>n$, and $\left\{u_{\lambda}\right\}$ be an approximate identity for $I$. Let $x \in \mathrm{QM}(A, I)$. If we define $x_{i j}=\left(e_{i}-e_{i-1}\right)^{1 / 2} x\left(e_{j}-e_{j-1}\right)^{1 / 2}\left(e_{0}=0\right)$, then $x_{i j} \in A \cap I^{* *}=I$. There is a subsequence $\left\{u_{n}\right\}$ of $\left\{u_{\lambda}\right\}$ such that

$$
\left\|\left(1-u_{i}\right) x_{i j}\right\|<1 / 2^{i+j}, \quad j \leq i, i=1,2, \ldots,
$$

and

$$
\left\|x_{i j}\left(1-u_{i}\right)\right\|<1 / 2^{i+j}, \quad i \leq j, j=1,2, \ldots .
$$

Define $u=\sum_{i=1}^{\infty}\left(e_{i}-e_{i-1}\right)^{1 / 2} u_{i}\left(e_{i}-e_{i}\right)^{1 / 2}$. By Lemma 2.2, $u \in I^{* *}$, it is then easy to check that $u \in \mathbf{M}(A, I)$. Define $a_{j i}=\left(1-e_{j+1}\right)\left(e_{i}-e_{i-1}\right)^{1 / 2}$, $b_{j}^{(1)}=\left(e_{j}-e_{j-1}\right)^{1+1 / 2}, b_{j}^{(2)}=\left(e_{j+1}-e_{j}\right)^{1 / 2}\left(e_{j}-e_{j-1}\right)$ and $b_{j}^{(3)}=$ $\left(e_{j-1}-e_{j-2}\right)^{1 / 2}\left(e_{j}-e_{j-1}\right)$. Since $a_{j i}=0$ if $i<j+1$, we have

$$
\begin{aligned}
& \left(1-e_{j+1}\right)[(1-u) x u]\left(e_{j}-e_{j-1}\right)=\sum_{i=j+1} a_{j i}\left(1-u_{i}\right) x_{i j} u_{j} b_{j}^{(1)} \\
& \quad+\sum_{i=j+1} a_{j i}\left(1-u_{i}\right) x_{i j+1} u_{j+1} b_{j}^{(2)}+\sum_{i=j+1} a_{j i}\left(1-u_{i}\right) x_{i j-1} u_{j-1} b_{j}^{(3)} .
\end{aligned}
$$

Thus

$$
\left\|\left(1-e_{j+1}\right)(1-u) x u\left(e_{j}-e_{j-1}\right)\right\|<1 / 2^{j-2}
$$

This implies

$$
\sum_{j=1}^{\infty}\left\|\left(1-e_{j+1}\right)(1-u) x u\left(e_{j}-e_{j-1}\right)\right\|<\infty .
$$

By Lemma 2.1, $(1-u) x u \in \mathbf{L M}(A)$. Similarly, $u x(1-u) \in \mathbf{R M}(A)$ and $(1-u) x(1-u) \in \operatorname{LM}(A) \cap \operatorname{RM}(A)$. For every $a \in A,(1-u) x u \cdot a \in A \cap I^{* *}=I$, $a \cdot u x(1-u) \in I$ and $a(1-u) x(1-u),(1-u) x(1-u) a \in I$. So $(1-u) x u \in$ $\operatorname{LM}(A, I), u x(1-u) \in \mathbf{R M}(A, I)$ and $(1-u) x(1-u) \in \mathbf{M}(A, I)$.

Now we need only show that $u x u \in \operatorname{LM}(A, I)+\operatorname{RM}(A, I)$.

(i) If $\mathrm{QM}(A)=\mathbf{L M}(A)+\operatorname{RM}(A)$, there are $y_{1} \in \mathbf{L M}(A)$ and $z_{1} \in \operatorname{RM}(A)$ such that $x=y_{1}+z_{1}$, so $u x u=u z_{1} u+u y_{1} u$. Since $u \in \mathbf{M}(A, I), y_{1} u, u a \in I$ for every $a \in A$. Hence $u y_{1} u \in \operatorname{LM}(A, I)$. Similarly, $u z_{1} u \in \operatorname{RM}(A, I)$.

(ii) If $\mathrm{QM}(I)=\mathrm{LM}(I)+\mathrm{RM}(I)$, there are $y_{2} \in \mathbf{L M}(I)$ and $z_{2} \in \operatorname{RM}(I)$ such that $x=y_{2}+z_{2}$, so $u x u=u y_{2} u+u z_{2} u$. One can easily check, as above, that $u y_{2} u \in \operatorname{LM}(A, I), u z_{2} u \in \operatorname{RM}(A, I)$. This completes the proof.

At this point, one may ask whether $\mathrm{QM}(A)=\mathrm{LM}(A)+\mathrm{RM}(A)$ implies $\mathrm{QM}(I)=\mathrm{LM}(I)+\mathrm{RM}(I)$. This turns out to be false, as we shall see in Example 8.1. However, we have the following "lifting" theorem. 
Theorem 3.3. Let $I$ be an ideal of a $\sigma$-unital $C^{*}$-algebra $A$, and suppose that $\mathrm{QM}(A / I)=\mathrm{LM}(A / I)+\mathrm{RM}(A / I)$ and $\mathrm{QM}(I)=\mathrm{LM}(I)+\mathrm{RM}(I)$. Then

$$
\mathrm{QM}(A)=\mathrm{LM}(A)+\mathrm{RM}(A) .
$$

Proof. Let $\phi: A \rightarrow A / I$ be the canonical homomorphsim and take $x \in \mathrm{QM}(A)$. So there is $\bar{y} \in \operatorname{LM}(A / I)$ and $\bar{z} \in \operatorname{RM}(A / I)$ such that $\phi^{* *}(x)=\bar{y}+\bar{z}$, where $\phi^{* *}$ is the extension of $\phi$ to $A^{* *}$. It follows from [6, 4.13] that there are $y_{1} \in \mathbf{L M}(A), z_{1} \in \mathbf{R M}(A)$ such that $\phi^{* *}\left(y_{1}\right)=\bar{y}$ and $\phi^{* *}\left(z_{1}\right)=\bar{z}_{1}$. Thus $\phi^{* *}\left(x-z_{1}-y_{1}\right)=0$. So we may assume that $x \in \operatorname{ker} \phi^{* *} \cap \mathrm{QM}(A)$, hence $x \in \mathrm{QM}(A, I)$. By Lemma 3.2 $x \in \operatorname{LM}(A, I)+\mathrm{RM}(A, I) \subset \operatorname{LM}(A)+\operatorname{RM}(A)$.

Let $K$ be the $C^{*}$-algebra of all compact operators on $l^{2}$.

Corollary 3.4. Let $A$ be a $C^{*}$-algebra such that $\mathrm{QM}(A \otimes K)=\mathrm{LM}(A \otimes K)+$ $\operatorname{RM}(A \otimes K)$. Then $\mathrm{QM}(\tilde{A} \otimes K)=\mathrm{LM}(\tilde{A} \otimes K)+\operatorname{RM}(\tilde{A} \otimes K)$.

Theorem 3.5. Let $A$ be a $C^{*}$-algebra such that $\mathrm{QM}(A \otimes K)=\mathrm{LM}(A \otimes K)+$ $\mathrm{RM}(A \otimes K)$ and let $B$ be a $\sigma$-unital $C^{*}$-subalgebra of $A$ such that $\mathrm{QM}(B)=$ $\mathrm{M}(B)$. Then

$$
\mathrm{QM}(B \otimes K)=\mathrm{LM}(B \otimes K)+\mathrm{RM}(B \otimes K) .
$$

Proof. By Corollary 3.4 we may assume that $A$ has an identity. Take $x \in$ $\mathrm{QM}(B \otimes K)_{\text {s.a. }}$ and let $\left\{e_{i j}\right\}$ be a set of matrix units for $K$. Then $x$ can be identified with an infinite matrix $\left(a_{i j}\right)$ which represents a bounded operator, where $a_{i j}$ is defined by $\left(1 \otimes e_{i i}\right) x\left(1 \otimes e_{j j}\right)=a_{i j} \otimes e_{i j}$. Clearly each $a_{i j} \in$ $\mathrm{QM}(B)=\mathbf{M}(B)$. i.e.

Let $\left\{u_{n}\right\}$ be an approximate identity of $B$ which is quasi-central for $\mathrm{M}(B)$,

$$
\lim \left\|u_{n} b-b u_{n}\right\|=0 \text { for all } b \in \mathbf{M}(B) .
$$

For every $i$, we have an integer $n_{i}$ such that

$$
\max _{k, j \leq i}\left[\left\|\left(1-u_{n_{i}}\right) u_{k}\right\| \cdot\left\|a_{i j}\right\|\right]<1 / 2^{2 i+1}
$$

and

$$
\left\|u_{n_{i}} a_{i j}-a_{i j} u_{n_{i}}\right\|<1 / 2^{i+j}, \quad i \geq j .
$$

Let $w=\left(b_{i j}\right)$, where $b_{i i}=u_{n_{i}}, b_{i j}=0$, if $i \neq j$. Clearly, $w$ is bounded and so is $w x=\left(u_{n_{i}} a_{i j}\right)$. Since $a_{i j} \in \mathbf{M}(B), u_{n_{i}} a_{i j} \in B \subset A$. We may view $w x$ as an element in $\mathrm{QM}(A \otimes K)$. It follows from $[6,4.20]$ or Theorem 2.3 that there exist $n_{1}<n_{2}<\cdots$ such that $L(w x)$ is bounded, where

$$
L(w x)=\sum_{k=1}^{\infty}\left(1-f_{n_{k}}\right) w x\left(f_{n_{k}}-f_{n_{k-1}}\right)
$$

and $f_{n}=\sum_{i=1}^{n} 1 \otimes e_{i i}$. Let $\sigma=-L(w x)+L(w x)^{*}$ and $y=x+\sigma$. Then $y$ is bounded and $\operatorname{Re} y=x$. Let $L(y)^{\prime}=\left(c_{i j}\right)$, where $c_{i j}=a_{i j}\left(1-u_{n_{i}}\right)$, if there is $k>l$ such that $n_{k-1}<i \leq n_{k}, n_{l-1}<j \leq n_{l}$, and $c_{i j}=0$ otherwise. Then 
$L(y)-L(y)^{\prime}=\left(d_{i j}\right)$ where $d_{i j}=a_{i j} u_{n_{i}}-u_{n_{i}} a_{i j}$ if there is $k>l$ such that $n_{k-1}<i \leq n_{k}, n_{l-1}<j \leq n_{l}$ and $d_{i j}=0$ otherwise. Since $\left\|d_{i j}\right\|<1 / 2^{i+j}$, $d_{i j} \in B$, we see that $L(y)-L(y)^{\prime} \in B \otimes K$. For every $k$,

$$
L(y)^{\prime} \sum_{i=1}^{k} u_{k} \otimes e_{i i} \in B \otimes K,
$$

because

$$
\max _{k, j \leq i}\left\|a_{i j}\right\| \cdot\left\|\left(1-u_{n_{i}}\right) u_{k}\right\|<1 / 2^{2 i+1} .
$$

Moreover $[y-L(y)] \cdot \sum_{i=1}^{k} u_{k} \otimes e_{i i} \in B \otimes K$. Hence

$$
y \cdot \sum_{i=1}^{k} u_{k} \otimes e_{i i} \in B \otimes K \quad \text { for all } k .
$$

Since $\left\{\sum_{i=1}^{k} u_{k} \otimes e_{i i}\right\}$ forms an approximate identity for $B \otimes K$, we conclude that $y \in \mathbf{L M}(A)$, so $x \in \mathbf{L M}(A)+\operatorname{RM}(A)$.

\section{A CONSTANT ASSOCIATED WITH THE EQUATION

$$
\mathrm{QM}(A)=\mathrm{LM}(A)+\mathrm{RM}(A)
$$

Definition 4.1. Let $A$ be a $C^{*}$-algebra such that $\mathrm{QM}(A)=\mathrm{LM}(A)+\operatorname{RM}(A)$. For every $x \in \mathrm{QM}(A)$, let

$$
\alpha(x)=\inf \{\|y\|: x=y+z, y \in \operatorname{LM}(A), z \in \operatorname{RM}(A)\} .
$$

Clearly $\alpha(x)<\infty$. Let $\alpha(A)=\sup _{\|x\| \leq 1} \alpha(x)$. To see that $\alpha(A)<\infty$, we consider the mapping $\phi: \mathrm{LM}(A) \rightarrow \mathrm{QM}(A)_{\text {s.a. }}$ defined by $\phi(x)=\left(x+x^{*}\right) / 2$. Then $\phi$ is a bounded real linear map from the real Banach space $\operatorname{LM}(A)$ onto the real Banach space $\mathrm{QM}(A)_{\text {s.a. }}$. By the open mapping theorem, $\phi$ is open. Thus the image of unit ball of $\operatorname{LM}(A)$ under $\phi$ contains a ball around the origin. It follows that $\alpha(A)<\infty$.

The following is an immediate consequence of Theorem 1.8.

Proposition 4.2. Let $A_{n}$ be $C^{*}$-algebras satisfying $\mathrm{QM}\left(A_{n}\right)=\mathrm{LM}\left(A_{n}\right)+$ $\operatorname{RM}\left(A_{n}\right)$ and $\alpha\left(A_{n}\right)<c$, for some $c>0$. Then

$$
\mathrm{QM}\left(\Sigma \oplus A_{n}\right)=\mathbf{L M}\left(\Sigma \oplus A_{n}\right)+\operatorname{RM}\left(\Sigma \oplus A_{n}\right)
$$

and $\sup _{n} \alpha\left(A_{n}\right) \leq \alpha\left(\Sigma \oplus A_{n}\right) \leq c$.

Lemma 4.3. Suppose that $A$ is a $\sigma$-unital $C^{*}$-algebra such that $\mathrm{QM}(A)=$ $\mathrm{LM}(A)+\operatorname{RM}(A)$. If $\left\{e_{n}\right\}$ is an approximate identity satisfying $e_{m} e_{n}=e_{n} e_{m}=$ $e_{n}$, if $m>n$, and $x \in \mathrm{QM}(A)$ with $x \leq 1$, then for every $\varepsilon>0$, there is $n_{1}<n_{2}<\cdots$ such that

$$
\left\|\sum_{k=1}^{\infty}\left(1-e_{n_{k}}\right) x\left(e_{n_{k}}-e_{n_{k-1}}\right)\right\| \leq 7 \alpha(A)+5+\varepsilon .
$$


156

HUAXIN LIN

Proof. Let $x=y+z$, where $y \in \operatorname{LM}(A), z \in \mathrm{RM}(A)$ and $\|y\| \leq \alpha(A)+$ $(1 / 21) \varepsilon$

As in the proof of Lemma 2.1, there exist $n_{1}<n_{2}<\cdots$ such that

$$
\sum_{k=1}^{\infty}\left\|\left(1-e_{n_{k+1}}\right) y\left(e_{n_{k}}-e_{n_{k-1}}\right)\right\|<\frac{\varepsilon}{3}
$$

and

$$
\sum_{k=1}^{\infty}\left\|\left(e_{n_{k}}-e_{n_{k-1}}\right) z\left(1-e_{n_{k+1}}\right)\right\|<\frac{\varepsilon}{3} .
$$

Define $z_{i k}=\left(e_{n_{i}}-e_{n_{i-1}}\right) z\left(e_{n_{k}}-e_{n_{k-1}}\right)$. We have

$$
\begin{gathered}
\left\|\sum_{k=1}^{\infty}\left(1-e_{n_{k}}\right) z\left(e_{n_{k}}-e_{n_{k-1}}\right)\right\|=\left\|z-\sum_{k=1}^{\infty} e_{n_{k}} z\left(e_{n_{k}}-e_{n_{k-1}}\right)\right\| \\
\leq\|z\|+\left\|\sum_{k=1}^{\infty} \sum_{i=1}^{k} z_{i k}\right\| \\
\leq\|z\|+\left\|\sum_{k=1}^{\infty} \sum_{i=1}^{k-2} z_{i k}\right\|+\left\|\sum_{k=1}^{\infty} z_{k-1 k}\right\|+\left\|\sum_{k=1}^{\infty} z_{k k}\right\| .
\end{gathered}
$$

For every large $N$

$$
\begin{aligned}
\left\|\sum_{k=1}^{N} \sum_{i=1}^{k-2} z_{i k}\right\| & =\left\|\sum_{i=1}^{N-2} \sum_{k=i+2}^{N} z_{i k}\right\| \\
& =\left\|\sum_{i=1}^{N-2}\left(e_{n_{i}}-e_{n_{i-1}}\right) z\left(e_{n_{N}}-e_{n_{i+1}}\right)\right\|<\frac{\varepsilon}{3} .
\end{aligned}
$$

Thus, by the proof of Lemma 2.2 ,

$$
\left\|\sum_{k=1}^{\infty}\left(1-e_{n_{k}}\right) z\left(e_{n_{k}}-e_{n_{k-1}}\right)\right\| \leq 5\|z\|+\frac{\varepsilon}{3} .
$$

Now we have

$$
\begin{aligned}
& \left\|\sum_{k=1}^{\infty}\left(1-e_{n_{k}}\right) x\left(e_{n_{k}}-e_{n_{k-1}}\right)\right\| \leq\left\|\sum_{k=1}^{\infty}\left(1-e_{n_{k+1}}\right) y\left(e_{n_{k}}-e_{n_{k-1}}\right)\right\| \\
& \quad+\left\|\sum_{k=1}^{\infty}\left(e_{n_{k+1}}-e_{n_{k}}\right) y\left(e_{n_{k}}-e_{n_{k-1}}\right)\right\| \\
& \quad+\left\|\sum_{k=1}^{\infty}\left(1-e_{n_{k}}\right) z\left(e_{n_{k}}-e_{n_{k-1}}\right)\right\| \\
& \quad<\frac{\varepsilon}{3}+2\|y\|+5\|z\|+\frac{\varepsilon}{3} \\
& \quad<\frac{\varepsilon}{3}+2 \alpha(A)+\frac{2}{21} \varepsilon+5 \alpha(A)+\frac{5}{21} \varepsilon+5+\frac{\varepsilon}{3} \\
& =7 \alpha(A)+5+\varepsilon .
\end{aligned}
$$


Remark 4.4. From the proof of Lemmas 2.2 and 4.3, we find that if the $e_{n}$ 's are projections, we will have

$$
\left\|\sum\left(1-e_{n_{k}}\right) x\left(e_{n_{k}}-e_{n_{k-1}}\right)\right\| \leq 4 \alpha(A)+3+\varepsilon .
$$

Lemma 4.5. Let $B$ be a $C^{*}$-algebra which has an approximate identity consisting of countably many projections. Suppose that $\mathrm{QM}(B)=\mathrm{M}(B)$ and $A=C(X) \otimes$ $B$, where $X$ is homeomorphic to one of the spaces $Y_{n}$ described in Definition 1.6. Then

$$
\mathrm{QM}(A)=\mathrm{LM}(A)+\mathrm{RM}(A) \text { and } \alpha(A) \leq \sqrt{n} .
$$

Proof. Let $x \in \mathrm{QM}(A)=C\left(X, \mathrm{QM}(B)_{\mathrm{Q} . S .}\right)$ and $\|x\| \leq 1$. Let $\left\{e_{k}\right\}$ be an approximate identity of $B$ consisting of projections. We use induction.

(1) If $n=1, Y_{1}=\{0\} \cup\{1 / m\}_{m=1}^{\infty}$.

For every $k$, there is an $N$ such that whenever $m \geq N$

$$
\left\|e_{k}(x(0)-x(1 / m)) e_{k}\right\|<1 / k .
$$

There is an integer $m_{0}$ such that for every $m \geq m_{0}$, there is a largest integer $k_{m}$ such that

$$
\left\|e_{k_{m}}(x(0)-x(1 / l)) e_{k_{m}}\right\| \leq 1 / k_{m} \text { for all } l \geq m .
$$

(In the trivial case $x(1 / m)=x(0)$ for all $m \geq m^{*}$, for some $m^{*}$, define $\left.e_{k_{m}}=e_{m}.\right)$ Hence $k_{m} \rightarrow \infty$, as $m \rightarrow \infty$ and $k_{m+1} \geq k_{m}$. Define $u(1 / m)=e_{1}$ if $^{\prime} m<m_{0}, u(1 / m)=e_{k_{m}}$ and $y(0)=x(0), y(1 / m)=u(1 / m) x(1 / m)$, $z(0)=0$ and $z(1 / m)=(1-u(1 / m)) x(1 / m)$. Then $x=y+z$. It is easy to check that for every $a$ and $b \in A, y(1 / m) a \rightarrow y(0) a$ and $b z(1 / m) \rightarrow b z(0)$. So $y \in \operatorname{LM}(A), z \in \operatorname{RM}(A)$ and

$$
\|y\|=\|u x\| \leq 1=\sqrt{1} .
$$

(2) Next we assume that Lemma 4.5 is true for all integers less than $n$. In particular, we can choose $y \in \operatorname{LM}(A)$ such that $\|y\| \leq \sqrt{k}$, where $k<n$.

Notice that $Y_{n}$ is the one-point compactification of the disjoint union of $Z_{i}$, where each $Z_{i}$ is homeomorphic to $Y_{n-1}$.

Let $x_{i}(t)=\left.x(t)\right|_{Z_{i}}$. There is an integer $i_{0}$ such that for every $i \geq i_{0}$, there is a largest integer $m_{i}$ such that $\left\|e_{m_{i}}\left[x(\infty)-x_{i}(t)\right] e_{m_{i}}\right\| \leq 1 / m_{i}$ for $t \in Z_{i}$. (In the case that $x_{i}(t) \equiv x(\infty)$ for all $i \geq i_{0}$, for some $i_{0}$, we define $e_{m_{i}}=e_{i}$.) Hence $m_{i} \rightarrow \infty$, as $i \rightarrow \infty$ and $m_{i+1} \geq m_{i}$. By the induction assumption, there are $y_{i} \in C\left(Z_{i}, \operatorname{LM}(B)_{\text {L.S. }}\right)$ and $z_{i} \in C\left(Z_{i}, \operatorname{RM}(B)_{\text {R.S. }}\right)$ such that $x_{i}=y_{i}+z_{i}$ and $\left\|y_{i}\right\| \leq \sqrt{n-1}$.

Define $y(t)=e_{m_{i}} x_{i}(t)+\left(1-e_{m_{i}}\right) y_{i}(t)\left(1-e_{m_{i}}\right)$ if $t \in Z_{i}, y(\infty)=x(\infty)$, $z(t)=\left(1-e_{m_{i}}\right) z_{i}(t)+\left(1-e_{m_{i}}\right) y_{i} e_{m_{i}}$, if $t \in Z_{i}$ and $z(\infty)=0$. Clearly, $x=y+z$ and $\left.y(t)\right|_{z_{i}} \in C\left(Z_{i}, \mathrm{LM}(B)_{\mathrm{L.S} .}\right)$ and $\left.z(t)\right|_{Z_{i}} \in C\left(Z_{i}, \operatorname{RM}(B)_{\mathrm{R} . S .}\right)$. Similarly to (1), one can check that $y(t) \in C\left(Y_{n}, \mathrm{LM}(B)_{\text {L.S. }}\right)=\mathrm{LM}(A)$ and 
$z(t) \in C\left(Y_{n}, \operatorname{RM}(B)_{\text {R.S. }}\right)=\operatorname{RM}(A)$. Hence $x \in \mathrm{LM}(A)+\operatorname{RM}(A)$. Moreover, let $B$ act on a Hilbert space $H$ and $f \in H$,

$$
\begin{aligned}
\|y(t) f\|^{2} & =\left\|e_{m_{i}} x_{i}(t) f\right\|^{2}+\left\|\left(1-e_{m_{i}}\right) y_{i}(t)\left(1-e_{m_{i}}\right) f\right\|^{2} \\
& \leq\|f\|^{2}+(n-1)\|f\|^{2}=n\|f\|^{2} .
\end{aligned}
$$

This implies that $\|y\| \leq \sqrt{n}$.

Lemma 4.6. Let $A=C_{0}(X, A(t), A)$ be a separable $C^{*}$-algebra, where $X$ is a countable, locally compact Hausdorff space with $\lambda(X)<\infty$ and $A(t)$ are $C^{*}$-algebras such that $\mathrm{QM}(A(t))=\mathrm{M}(A(t))$. Then $\mathrm{QM}(A)=\mathrm{LM}(A)+\mathrm{RM}(A)$. Proof. Let $I_{k}=\left\{f \in A, f(t)=0\right.$, if $\left.t \in X_{[k]}\right\}$ (cf. Definition 1.4). By Proposition 4.2, and Theorem 3.3, we can easily prove the lemma by induction. Remark 4.6. Define $f\left(e^{i \theta}\right)=i \theta / \pi \quad(-\pi<\theta \leq \pi)$ and let $a_{n}(n \in \mathbf{Z})$ be its Fourier coefficients. Then $a_{n}=(-1)^{n+1} / n \pi \quad(n \neq 0), a_{0}=0$. But $\sum_{n \in \mathrm{Z}} a_{|n|} e^{i n \theta}$ is the Fourier series of the $L^{2}$ function $2 \pi^{-1} \log \left|1+e^{i \theta}\right|$ which is not in $L^{\infty}(T)$. ( $T$ denoting the unit circle.) Thus the matrix $\left(a_{i-j}\right)$ represents an operator on $l^{2}$ of norm 1 . But the lower triangle of the matrix is not bounded. Let $L_{n}=\left(b_{i j}\right)$, where $b_{i j}=\sqrt{-1} a_{i-j}$ if $i \geq j$ and $i \leq n, b_{i j}=0$ if $i>n$, or $j>i$.

Let $g \in l^{2}, g=\left(d_{j}\right), d_{j}=(-1)^{j} 1 / \sqrt{n}^{1 / 2}, j \leq n, d_{j}=0, j>n$. Then $\|g\|_{2}=1$ and

$$
\begin{aligned}
\left\|L_{n} g\right\|^{2} & =\frac{1}{\pi^{2} n} \sum_{k=1}^{n}\left(\sum_{j=1}^{k-1} \frac{1}{j}\right)^{2} \geq \frac{1}{\pi^{2} n} \sum_{k=1}^{n}(\log K)^{2} \\
& \geq \frac{1}{\pi^{2} n}[n \log n(\log n-2)] \geq \frac{1}{4 \pi^{2}}(\log n)^{2}
\end{aligned}
$$

if $n$ is large enough $(n>15)$. We conclude that $\left\|L_{n}\right\| \geq(1 / 2 \pi) \log n$ when $n$ is large.

Lemma 4.7. Let $A_{n}=C\left(Y_{n}\right) \otimes K$. Then

$$
\frac{1}{9 \pi} \log n \leq \alpha\left(A_{n}\right) \leq \sqrt{n}
$$

when $n$ is large enough $(n>20)$.

Proof. It follows from Lemma 4.5 that $\mathrm{QM}\left(A_{n}\right)=\mathrm{LM}\left(A_{n}\right)+\operatorname{RM}\left(A_{n}\right)$ and $\alpha\left(A_{n}\right) \leq \sqrt{n}$.

For every sequence $\left\{n_{k}\right\}, n_{1}<n_{2}<\cdots$, define the operator $\alpha\left(\left\{n_{k}\right\}\right)=\left(t_{i j}\right)$ where

$$
t_{i j}= \begin{cases}\sqrt{-1}\left(a_{k-l}\right), & \text { if } i=n_{k}, j=n_{l}, \\ 0 & \text { otherwise }\end{cases}
$$

and $a_{n}=(-1)^{n+1} / n \pi, n \neq 0, n \in \mathbf{Z}, a_{0}=0$. 
Then $\alpha\left(\left\{n_{k}\right\}\right)$ is selfadjoint and $\left\|\alpha\left(\left\{n_{k}\right\}\right)\right\| \leq 1$. For every $n$, define $\alpha_{n}\left(\left\{n_{k}\right\}\right)=\left(t_{i j}^{\prime}\right)$, where

$$
t_{i j}^{\prime}= \begin{cases}\sqrt{-1}\left(a_{k-l}\right), & \text { if } i=n_{k}, j=n_{l} \text { and } k, l \leq n, \\ 0 & \text { otherwise. }\end{cases}
$$

Let $S_{n}=\left\{a_{n}\left(\left\{n_{k}\right\}\right),\left\{n_{k}\right\} \subset \mathbf{N}\right\}$, so that each $S_{n}$ is countable $(n \in \mathbf{N})$. We claim that the cluster points of $S_{n}$ are

$$
\left\{\alpha_{j}\left(\left\{n_{k}\right\}\right), j \leq n-1,\left\{n_{k}\right\} \subset N\right\}=\bigcup_{j=0}^{n-1} S_{j}
$$

(in the weak operator topology).

Let $p_{m}=\left(\varepsilon_{i j}\right)$ where $\varepsilon_{i i}=1$, if $i \leq m, \varepsilon_{i j}=0$, if $i \neq j$ or $i>m$.

Let $\beta \in \bigcup_{j=0}^{n-1} S_{j}$, say $\beta=\alpha_{j}\left(\left\{n_{k}\right\}\right)$ for some $j \leq n-1$ and $\left\{n_{k}\right\} \subset \mathbf{N}$. Define $n_{k}^{(s)}=n_{k}$ if $k \leq j, n_{k}^{(s)}=n_{k}+k+s$, if $k>j$. For every $m$, if $s>m$, $p_{m}\left[\beta-\alpha_{n}\left(\left\{n_{k}^{(s)}\right\}\right)\right] p_{m}=0$. This implies that $\alpha_{n}\left(\left\{n_{k}^{(s)}\right\}\right) \rightarrow \beta$ weakly as $s \rightarrow \infty$.

Next let $\alpha_{n}\left(\left\{m_{k}^{(s)}\right\}\right) \rightarrow \beta$ weakly as $s \rightarrow \infty$. Since for every $i$ there are only finitely many different elements $p_{i} \alpha_{n}\left(\left\{m_{k}^{(s)}\right\}\right) p_{i}$, we see that

$$
p_{i}\left[\alpha_{n}\left(m_{k}^{(s)}\right)-\beta\right] p_{i}=0,
$$

when $s$ is large. Thus we conclude that

$$
\beta \in\left\{\alpha_{j}\left(\left\{n_{k}\right\}\right), j \leq n-1,\left\{n_{k}\right\} \subset N\right\}=\bigcup_{j=0}^{n-1} S_{j}
$$

unless $\alpha_{n}\left\{n_{k}^{(s)}\right\}=\beta$ for $s \geq s_{0}$ for some $s_{0}$. This establishes the claim.

By induction, we have $\left(S_{n}\right)^{\bar{w}}=\bigcup_{j=1}^{n} S_{j}$ (where " $\bar{w}$ " means the weak closure) and $\left(S_{n}\right)_{[i]}^{\bar{w}}=\bigcup_{j=0}^{n-i} S_{j}$. Hence $\lambda\left(S_{n}^{\bar{w}}\right)=n$. It is also clear, by a similar argument as the above, that every sequence of $S_{n}$ has a convergent subsequence. So $S_{n}^{\bar{w}}$ is compact.

The weak operator topology on bounded subsets of $B\left(l^{2}\right)$ coincides with the quasi-strict topology $B\left(l^{2}\right)=\mathrm{QM}(K)$, so we define a continuous mapping $F_{n}$ from $Y_{n}$ onto $S_{n}^{\bar{w}}$, then $F_{n} \in C\left(Y_{n}, \mathrm{QM}(K)_{\mathrm{Q} . \mathrm{S} .}\right)=\mathrm{QM}\left(A_{n}\right)$. The existence of $F_{n}$ comes from Theorem 1.7. Now let $\left\{e_{i j}\right\}$ be a set of matrix units for $K$ and $f_{m}=\sum_{i=1}^{m} 1 \otimes e_{i i}\left(f_{m}\right.$ can be identified with a constant function: $\left.Y_{n} \rightarrow P_{m} \in K\right)$. Then $\left\{f_{m}\right\}$ forms an approximate identity for $A$. For every $n_{1}<n_{2}<\cdots$, we see clearly, by the construction of $S_{n}^{\bar{w}}$, that

$$
\left\|\sum_{k=1}^{\infty}\left(1-f_{n_{k}}\right) F_{n}\left(f_{n_{k}}-f_{n_{k-1}}\right)\right\| \geq\left\|L_{n}(\alpha)\right\| \geq \frac{1}{2 \pi} \log n,
$$

if $n$ is large enough $(n>15)$. 
Combining this and Remark 4.4, we have that $\alpha\left(A_{N}\right) \geq \frac{1}{9 \pi} \log n$, if $n$ is large enough $(n>20)$.

We shall see in Example 8.1 that $\mathrm{M}(A / I)=\mathrm{QM}(A / I)$ and $\mathrm{M}(I)=\mathrm{QM}(I)$ does not imply $\mathrm{QM}(A)=\mathrm{M}(A)$ even for very simple algebras. Theorem 3.3 shows that if moreover $A$ is $\sigma$-unital, we do have $\mathrm{QM}(A)=\operatorname{LM}(A)+\operatorname{RM}(A)$. Hence the only significance of the following proposition is the estimate of $\alpha(A)$.

Proposition 4.8. Let $A$ be a $\sigma$-unital $C^{*}$-algebra and $I$ an ideal of $A$. Suppose $\mathrm{M}(A / I)=\mathrm{QM}(A / I)$ and $\mathrm{M}(I)=\mathrm{QM}(I)$. Then for every $x \in \mathrm{QM}(A)$, there are $y \in \operatorname{LM}(A)$ and $z \in \operatorname{RM}(A)$ such that $x=y+z,\|y\| \leq\|x\|$ and $\|z\| \leq\|x\|$, hence $\alpha(A) \leq 1$.

Proof. Let $\phi: A \rightarrow A / I$ be the canonical homomorphism, $\phi^{* *}$ be its extension to $A^{* *}$. So $\phi(x) \in \mathrm{QM}(A / I)=\mathrm{M}(A / I)$. By [26], there is $x^{\prime} \in \mathrm{M}(A)$ such that $\phi\left(x^{\prime}\right)=\phi(x)$ and $x_{1}=x-x^{\prime} \in \operatorname{ker} \phi^{* *} \cap \mathrm{QM}(A)$. Thus $x_{1} \in \mathrm{QM}(A, I)$. Let $\left\{e_{i}\right\}$ be an approximate identity for $A$ satisfying $e_{i} e_{j}=e_{i} e_{j}=e_{i}$, if $i<j$, and put $x_{i j}=\left(e_{i}-e_{i-i}\right)^{1 / 2} x\left(e_{j}-e_{j-j}\right)^{1 / 2}$. Then $x_{i j} \in I$. Suppose that $\left\{u_{\lambda}\right\}$ is an approximate identity for $I$. There is a subsequence $\left\{u_{\lambda_{n}}\right\}$ of $\left\{u_{\lambda}\right\}$ such that

$$
\left\|\left(1-u_{\lambda_{i}}\right) x_{i j}\right\|<\frac{1}{2^{i+j}}, \quad i \geq j, i=1,2, \ldots .
$$

If $u=\sum_{i=1}^{\infty}\left(e_{i}-e_{i-1}\right)^{1 / 2} u_{\lambda_{i}}\left(e_{i}-e_{i-1}\right)^{1 / 2}$, then $u \in \mathbf{M}(A, I)$. As in the proof of Lemma 3.2 we have

$$
\sum_{i}\left\|\left(1-e_{i+1}\right)(1-u) x_{1}\left(e_{i}-e_{i-1}\right)\right\|<\infty .
$$

Hence $(1-u) x_{1} \in \operatorname{LM}(A)$ by Lemma 2.1 .

Let $y=(1-u) x=(1-u) x^{\prime}+(1-u) x_{1}$ and put $z=u x=u x_{1}+u x^{\prime}$. Since $u \in \mathbf{M}(A, I)$, we see that $u \in \mathbf{L M}(A)$ and $u x^{\prime} \in \mathbf{M}(A)$. Since $\mathbf{M}(I)=$ $\mathrm{QM}(I), x_{1} \in \mathrm{M}(I)$. For every $a \in A$, au $\in I$, so $a u x_{1} \in I \subset A$. This implies that $u x_{1} \in \mathbf{R M}(A)$. Hence $z \in \mathbf{R M}(A)$ since $0 \leq u \leq 1$. We have $\|y\|=\|(1-u) x\| \leq\|x\|$ and $\|z\|=\|u x\| \leq\|x\|$. Thus $\alpha(A) \leq 1$.

\section{THE SPECTRUM OF AN ELEMENT IN A SCATTERED $C^{*}$-ALGEBRA}

In this section, we shall discuss the relationship between the spectrum of a single element in a scattered $C^{*}$-algebra $A$ and the spectrum of the algebra $A$.

Jensen [13] defined a $C^{*}$-algebra to be scattered if every state on the algebra is atomic. He showed [14] that a $C^{*}$-algebra is scattered if and only if it is type I and has scattered spectrum $\hat{A}$. He also showed [14] that a $C^{*}$-algebra is scattered if and only if it has a composition series with elementary quotients.

We recall that a $C^{*}$-algebra $A$ is $\mathrm{AF}$ (approximately finite-dimensional) if for each $\varepsilon>0$ and $a_{1}, a_{2}, \ldots, a_{n} \in A$ there is a $C^{*}$-subalgebra $B$ of $A$ and $b_{1}, b_{2}, \ldots, b_{n} \in B$ such that $B$ is of finite dimension and $\left\|a_{i}-b_{i}\right\|<\varepsilon$, for all $i=1,2, \ldots, n$. 
Lemma 5.1. Every scattered $C^{*}$-algebra $A$ is $A F$.

Proof. Supose that $A$ has a series of ideals $0=I_{0} \subset I_{1} \subset I_{2} \subset \cdots \subset I_{\alpha} \subset \cdots \subset$ $I_{\lambda}=A$, where each $I_{\beta+1} / I_{\beta}$ is an elementary $C^{*}$-algebra and $I_{\alpha}=\left(\bigcup_{\beta<\alpha} I_{\beta}\right)^{-}$ for each limit ordinal $\alpha$. We prove the lemma by induction on $\lambda$. Assume Lemma 5.1 is true for all $\lambda<\lambda_{0}$.

If $\lambda_{0}$ is not a limit ordinal, $I_{\lambda_{0}} / I_{\lambda_{0}-1}$ is an elementary $C^{*}$-algebra, hence $I_{\lambda_{0}} / I_{\lambda_{0}-1}$ is an AF-algebra. By the induction hypothesis $I_{\lambda_{0}-1}$ is also an AFalgebra. It follows from [11] that $A$ is an AF-algebra.

If $\lambda_{0}$ is a limit ordinal, $A$ is the norm closure of $\bigcup_{\lambda<\lambda_{0}} I_{\lambda}$. For each $\varepsilon>0$ and $a_{1}, a_{2}, \ldots, a_{n} \in A$, there is $\lambda<\lambda_{0}$ and $b_{1}^{\prime}, b_{2}^{\prime}, \ldots, b_{n}^{\prime} \in I_{\lambda}$ such that

$$
\left\|a_{i}-b_{i}\right\|<\varepsilon / 2, \quad i=1,2, \ldots, n .
$$

Since, by hypothesis, $I_{\lambda}$ is an AF-algebra, there is a $C^{*}$-subalgebra $B$ of $I_{\lambda}$ and $b_{1}, b_{2}, \ldots, b_{n} \in B$ such that $B$ is of finite dimension and

$$
\left\|b_{i}^{\prime}-b_{i}\right\|<\varepsilon / 2, \quad i=1,2, \ldots, n .
$$

Hence $\left\|b_{i}-a_{i}\right\|<\varepsilon, i=1,2, \ldots, n$. So $A$ is an AF-algebra.

Lemma 5.2. Let $A$ be a scattered $C^{*}$-algebra. If $\lambda(\widehat{A})=\alpha$, then for every $a \in A_{\text {s.a. }}$, we have $\lambda[\sigma(a)] \leq \alpha+1$. If $\alpha$ is a limit ordinal and $\widehat{A}_{[\alpha]}=\varnothing$, then $\lambda[\sigma(a)] \leq \alpha$.

Proof. Let $I_{i}=\left\{x \in A ; \pi(x)=0, \forall \pi \in \widehat{A}_{[i]}\right\}_{i \leq \alpha}$. Suppose that $\alpha \in A_{\text {s.a. }}$. Let $B$ be the $C^{*}$-algebra generated by $a$. Define $J_{i}=B \cap I_{i}$. Clearly, since $I_{i+1} / I_{i}$ and $A / I_{\alpha}$ are dual $C^{*}$-algebras $[12,4.7 .20]$, so are $J_{i+1} / J_{i}$ and $B / J_{\alpha}$. Thus $\widehat{B}$ is the union of closed subsets $X_{i}$ satisfying $X_{i} \supset X_{i+1}, X_{i+1} \subset\left(X_{i}\right)_{[1]}$ and $X_{i+1} \backslash X_{i}$ is discrete, $i<\alpha$. If $\widehat{A}_{[\alpha]}=\varnothing, A=I_{\alpha}$. Hence $X_{\alpha}=\varnothing$. Since $B=C_{0}(\widehat{B})$, it is clear that $\lambda(\widehat{B}) \leq \alpha$ and if $\widehat{A}_{[\alpha]}=\varnothing, \widehat{B}_{[\alpha]}=\varnothing$. Thus $\lambda(\sigma(a)) \leq \alpha+1$ and if $\hat{A}_{[\alpha]}=\varnothing, \lambda(\sigma(a)) \leq \alpha$.

Lemma 5.3. Let $A$ be a scattered $C^{*}$-algebra. Suppose that $\lambda(\widehat{A})=\alpha, I_{\beta}=$ $\left\{x \in A ; \pi(x)=0, \pi \in \widehat{A}_{[\beta]}\right\}$. Then $I_{\beta+1} / I_{\beta}$ is of infinite dimension, if $\beta<\alpha$.

Proof. We shall use the facts that $A$ is of type I and $\beta+1 \leq \alpha$.

Let $J_{\beta+1}=I_{\beta+1} / I_{\beta}$. If $\widehat{J}_{\beta+1}$ is an infinite set, the result is clear. We may assume therefore that $\widehat{J}_{\beta+1}=\left\{\tilde{\pi}_{1}, \tilde{\pi}_{2}, \ldots, \tilde{\pi}_{m}\right\}$. Let $\pi_{i}$ be an irreducible representation of $A$ corresponding to $\tilde{\pi}_{i}$. We have $\widehat{A}_{[\beta]}=\bigcup_{i=1}^{m}\left\{\pi_{i}\right\}^{-}$. Since $\widehat{A}_{[\beta+1]} \neq \varnothing$, there is $\pi \in \widehat{A}_{[\beta+1]}$ and hence there is $i \leq m$ such that $\operatorname{ker} \pi_{i} \subset$ $\operatorname{ker} \pi$. This implies that $\pi_{i}(A)$ must be infinite dimensional. Hence $\pi_{i}\left(I_{\beta+1}\right) \supset$ $K\left(H_{\pi_{i}}\right)$ (the compact operators on $H_{\pi_{i}}$ ), where $\operatorname{dim} H_{\pi}=\infty$. Since $\pi_{i}\left(I_{\beta}\right)=$ 0 , we conclude that $J_{\beta+1}$ is of infinite dimension.

Theorem 5.4. Let $A$ be a scattered $C^{*}$-algebra with $\lambda(\widehat{A})=\alpha$. Then

(i) For every $a \in A_{\text {s.a. }} \quad \lambda(\sigma(a)) \leq \alpha+1$. 
(ii) If $\alpha$ is not a limit ordinal, there is $a \in A_{\text {s.a. }}$ such that $\lambda(\sigma(a)) \geq \alpha$.

(iii) If $\alpha$ is not a limit ordinal, then there is $a \in A_{\text {s.a. }}$ such that $\lambda(\sigma(a))=$ $\alpha+1$ if and only if $A / I_{\alpha}$ is of infinite dimension, where $I_{\alpha}=\{x \in$ $\left.A ; \pi(x)=0, \pi \in \widehat{A}_{[\alpha]}\right\}$.

(iv) If $\alpha$ is a limit ordinal and $A / I_{\alpha}$ is of finite dimension (or zero), then for every $a \in A_{\text {s.a. }}, \lambda(\sigma(a)) \leq \alpha$. Moreover, for every $\beta<\alpha$, there is $a \in A_{\text {s.a. }}$ such that $\lambda(\sigma(a))>\beta$.

(v) If $\alpha$ is a limit ordinal such that $\alpha=\lim \beta_{n}\left(\beta_{n}<\alpha\right)$ and $A / I_{\alpha}$ is of infinite dimension, then there is $a \in A_{\text {s.a. }}$ such that $\lambda(\sigma(a)) \geq \alpha$.

Proof. We shall use induction.

Assume the theorem is true for all $\beta<\alpha$.

(i) is the same as Lemma 5.4 .

(ii) If $\alpha$ is not a limit ordinal, by Lemma 5.5, $I_{\alpha} / I_{\alpha-1}$ is of infinite dimension. By the induction hypothesis for (iii), there is $a \in I_{\alpha}$ such that $a$ is selfadjoint and $\lambda(\sigma(a)) \geq(\alpha-1)+1=\alpha$.

(iii) If $A / I_{\alpha}$ is of finite dimension, $a \in A_{\text {s.a. }}$, then there is a polynomial $p(t)$ $(p \neq 0)$ such that $p(a) \in I_{\alpha}$. By the induction hypothesis $\lambda(\sigma(p(a))) \leq \alpha$, since $\lambda\left(\widehat{I}_{\alpha}\right)=\alpha-1$. By the spectral mapping theorem, one sees easily that $\lambda(\sigma(a)) \leq \alpha$.

If $A / I_{\alpha}$ is of infinite dimension, there is a sequence of mutually orthogonal projections $\bar{p}_{n} \in A / I_{\alpha}, \bar{p}_{n} \neq 0$. Let $\phi: A \rightarrow A / I_{\alpha}$ be the canonical homomorphism. Since $I_{\alpha}$ is an AF-algebra, by the projection lifting theorem [4], there is $p_{1} \in A$ such that $\phi\left(p_{1}\right)=\bar{p}_{1}$. Using the projection lifting theorem on $\left(1-p_{1}\right) A\left(1-p_{1}\right) / I_{\alpha} \cap\left(1-p_{1}\right) A\left(1-p_{1}\right) \cong\left(1-\bar{p}_{1}\right)(A / I)\left(1-\bar{p}_{1}\right)$, and continuing, we construct a sequence of mutually orthogonal projections $\left\{p_{n}\right\} \subset A$ such that $\pi\left(p_{n}\right)=\bar{p}_{n}$. Since there is $\pi \in \widehat{A}_{[\alpha]}$ such that $\pi\left(p_{n}\right) \neq 0$, we have $\pi \in \widehat{A} \backslash \operatorname{hull}\left(p_{n} A p_{n}\right)$. It follows from the fact that $p_{n} A p_{n}$ is a hereditary $C^{*}$ subalgebra of $A$ that $\left(p_{n} A p_{n}\right)^{\wedge}$ is homeomorphic to $\widehat{A} \backslash \operatorname{hull}\left(p_{n} A p_{n}\right)$. Since $\left(\widehat{A} \backslash \operatorname{hull}\left(p_{n} A p_{n}\right)\right)$ is open and $\widehat{A}_{[\alpha]} \cap\left(\widehat{A} \backslash \operatorname{hull}\left(p_{n} A p_{n}\right)\right) \neq \varnothing, \lambda\left(\left(p_{n} A p_{n}\right)^{\wedge}\right)=\alpha$. By (ii), there are $a_{n} \in p_{n} A p_{n}, a_{n}=a_{n}^{*},\left\|a_{n}\right\| \leq 1$ and $\lambda\left(\sigma\left(a_{n}\right)\right) \geq \alpha$. Taking $a_{n}^{2}$, if necessary, we may assume that $0 \leq a_{n} \leq 1$. Define

$$
a=\sum_{n=1}^{\infty} \frac{1}{2^{n}}\left(p_{n}+a_{n}\right) ;
$$

then $a$ is selfadjoint and $\lambda(\sigma(a))=\alpha+1$.

(iv) Assume that $\alpha$ is a limit ordinal and $A / I_{\alpha}$ is of finite dimension. If $a \in I_{\alpha}, a=a^{*}$, then by Lemma $5.2 \lambda(\sigma(a)) \leq \alpha$. For every $a \in A_{\text {s.a. }}$, there is a polynomial $p(t) \quad(p(t) \neq 0)$ such that $p(a) \in I_{\alpha}$. Hence $\lambda(\sigma(p(a))) \leq \alpha$. By the spectral mapping theorem, one can see easily that $\lambda(\sigma(a)) \leq \alpha$. For each $\beta<\alpha$, consider $I_{\beta+1} \subset A$. By the induction hypothesis, there is $a \in A_{\text {s.a. }}$ such that $\lambda(\sigma(a)) \geq \beta$. 
(v) If $\alpha$ is a limit ordinal such that $\alpha=\lim \beta_{n}, \beta_{n}<\alpha$, and $A / I_{\alpha}$ is of infinite dimension, then, as in the proof of (iii), $A$ contains a sequence of mutually orthogonal projections $\left\{q_{n}\right\}$ such that $\lambda\left[\left(q_{n} A q_{n}\right)^{\wedge}\right]=\alpha$. By (iv), there are $a_{n} \in q_{n} A q_{n}, 0 \leq a_{n} \leq 1$ such that, $\lambda\left(\sigma\left(a_{n}\right)\right) \geq \beta_{n}$. Define

$$
a=\sum_{n=1}^{\infty} \frac{1}{2^{n}}\left(q_{n}+a_{n}\right) \text {. }
$$

Clearly $a \in A_{\text {s.a. }}$ and $\lambda(\sigma(a)) \geq \alpha$.

The proof is complete.

\section{QUASI-MULTIPLIERS OF STABLE $C^{*}$-ALGEBRAS}

Lemma 6.1. Let $A$ be a separable scattered $C^{*}$-algebra with $\lambda(\widehat{A})<\infty$. Then $\mathrm{QM}(A)=\mathrm{LM}(A)+\mathrm{RM}(A)$.

Proof. Let $I_{i}=\left\{a \in A, \pi(a)=0, \forall \pi \in \widehat{A}_{[i]}\right\}$. Then $\{0\}=I_{0} \subset I_{1} \subset I_{2} \subset \cdots \subset$ $I_{n} \subset A, n=\lambda(\widehat{A})$, and $I_{i} / I_{i-1}$ and $A / I_{n}$ are separable dual $C^{*}$-algebras. Since $A$ and $I_{i}$ are $\sigma$-unital and $\mathrm{M}\left(I_{i} / I_{i-1}\right)=\mathrm{QM}\left(I_{i} / I_{i-1}\right), \mathrm{M}\left(A / I_{n}\right)=\mathrm{QM}\left(A / I_{n}\right)$, by Theorem 3.3 and induction; $\mathrm{QM}(A)=\mathrm{LM}(A)+\mathrm{RM}(A)$.

Corollary 6.2. Let $A$ be a separable scattered $C^{*}$-algebra with $\lambda(\widehat{A})<\infty$. Then $\mathrm{QM}(A \otimes K)=\mathrm{LM}(A \otimes K)+\mathrm{RM}(A \otimes K)$.

Theorem 6.3. Let $A$ be a separable $C^{*}$-algebra. Then $\mathrm{QM}(A \otimes K)=$ $\mathrm{LM}(A \otimes K)+\operatorname{RM}(A \otimes K)$ if and only if $A$ is scattered and $\lambda(\widehat{A})<\infty$.

Proof. By Corollary 6.2, we need only show the "only if" part. So we assume that $\mathrm{QM}(A \otimes K)=\mathrm{LM}(A \otimes K)+\mathrm{RM}(A \otimes K)$. It follows from Corollary 3.4 that we may assume that $A$ has an identity. It follows from $[6,4.23]$ that $A$ is scattered. If $\lambda(\widehat{A})$ is not finite, by Theorem 5.4, for every integer $m>0$, there is $a \in A_{\text {s.a. }}$ such that $\lambda(\sigma(a))=m$. Let $B$ be the $C^{*}$-algebra generated by $a$ and 1. It follows from the proof of Proposition 2.4 that $\mathrm{QM}(B \otimes K) \subset$ $\mathrm{QM}(A \otimes K)$ and $A \otimes K$ and $B \otimes K$ share a common approximate identity $f_{n}=\sum_{i=1}^{n} 1 \otimes e_{i j}$, where $\left\{e_{i j}\right\}$ is a set matrix units for $K$. By Lemma 2.16, there is $F \in \mathrm{QM}(B \otimes K) \subset \mathrm{QM}(A \otimes K)$ such that for every $\left\{n_{k}\right\}, n_{1}<n_{2}<\cdots$,

$$
\left\|\sum_{k=1}^{\infty}\left(1-f_{n_{k}}\right) F\left(f_{n_{k}}-f_{n_{k-1}}\right)\right\| \geq \frac{1}{2 \pi} \log m,
$$

if $m$ is large enough. It follows from Lemma 4.3 and Remark 4.4 that

$$
\alpha(A \otimes K) \geq \frac{1}{9 \pi} \log m
$$

for $m$ large enough. Hence $\alpha(A \otimes K)=\infty$, a contradiction.

Corollary 6.4. Let $A$ be a separable $C^{*}$-algebra. Then $\mathrm{QM}(A \otimes K)=$ $\mathbf{L M}(A \otimes K)+\operatorname{RM}(A \otimes K)$ if and only if there is an integer $m>0$ such that for every $a \in A_{\text {s.a. }}, \sigma(a)$ is countable and $\lambda(\sigma(a)) \leq m$. 
Proof. It is an immediate consequence of Theorem 5.1, [14, Theorem 2.2] and Theorem 6.2.

Corollary 6.5. Let $A$ be a separable stable $C^{*}$-algebra. Then $\mathrm{QM}(A)=\mathrm{LM}(A)$ $+\operatorname{RM}(A)$ if and only if $A$ is scattered and $\lambda(\widehat{A})<\infty$.

\section{7. $C^{*}$-ALGEBRAS WITH FINITE DIMENSIONAL IRREDUCIBLE REPRESENTATIONS}

In this section we shall consider $C^{*}$-algebras whose irreducible representations are finite dimensional. Let $M_{n}$ denote the $C^{*}$-algebra of all complex $n \times n$ matrices. If $A$ is a $C^{*}$-algebra whose irreducible representations are finite dimensional and $\widehat{A}$ is Hausdorff, then by [9, Theorem 10.54], $A=C_{0}\left(\widehat{A}, M_{n(t)}, A\right)$. If $A=C_{0}\left(\widehat{A}, M_{n(t)}, A\right)$ is locally trivial, one can easily show by Theorem 1.3 that $\mathrm{QM}(A)=\mathrm{M}(A)$. However, even if $\hat{A}$ is countable and Hausdorff, $\mathrm{QM}(A) \neq \mathrm{LM}(A)+\mathrm{RM}(A)$, in general.

Proposition 7.1. There is a $C^{*}$-algebra $A$ such that all of its irreducible representations are finite dimensional, $\hat{A}$ is a countable locally compact Hausdorff space, and $\mathrm{QM}(A) \neq \mathrm{LM}(A)+\mathrm{RM}(A)$.

Proof. Keep the notations in the proof of Lemma 4.5. Let $P^{(n)}(t)$ be the range projection of $F_{n}(t)$. By the proof of Lemma 4.5, it is clear that $P^{(n)}(t)$ is a weakly continuous mapping from $Y_{n}$ to $K$. Since $P^{(n)}(t)$ is bounded, we conclude that $P^{(n)}(t) \in \mathrm{QM}\left(C\left(Y_{n}, K\right)\right)$.

Let $X$ be the disjoint union of $Y_{n}, n=1,2, \ldots$. Define

$$
B_{0}=\left\{x \in C_{0}(X, K): x(t)=P^{(n)}(t) x(t) P^{(n)}(t) ; \forall t \in Y_{n}\right\} .
$$

Clearly, $B_{0}$ is a *algebra. Let $M_{n}(t)=P^{(n)}(t) K P^{(n)}(t)$. Then each $M_{n}(t)$ is isomorphic to some $M_{k}$. We define $A=C_{0}\left(X, M_{n}(t), B_{0}\right) . A$ is a $C^{*}$-algebra all of whose irreducible representations are of finite dimension and $\widehat{A}=X$, a countable, locally compact Hausdorff space. Define

$$
q_{k}(t)=P^{(n)}(t) \sum_{i=1}^{k} 1 \otimes e_{i i} P^{(n)}(t), \quad \text { if } k \geq m(n) \text { and } t \in Y_{n},
$$

and $q_{k}(t)=0$, if $k<m(n)$ and $t \in Y_{n}$, where $m(n)$ is the largest integer such that

$$
\left\|L_{m(n)}(\alpha)\right\| \leq[\log (n+1)]^{1 / 4} .
$$

Since $m(n) \rightarrow \infty, q_{k}(t) \in A$. Moreover, $\left\{q_{k}(t)\right\}$ forms an approximate identity for $A$.

Define $F(t)=F_{n}(t)$, if $t \in Y_{n}, n=1,2, \ldots$, so that $F(t) \in \mathrm{QM}(A)$. By the proof of Lemma 4.5 we have for every $\left\{n_{k}\right\} \subset \mathbf{N}$, if $n$ is large, that

$$
\begin{aligned}
& \left\|\sum\left(1-q_{n_{k}}\right) F\left(q_{n_{k}}-q_{n_{k-1}}\right)\right\| \\
& \quad \geq \frac{1}{2 \pi} \log n-[\log (n+1)]^{1 / 4} \quad(\rightarrow \infty, \text { as } n \rightarrow \infty) .
\end{aligned}
$$

Thus $F \notin \mathrm{LM}(A)+\mathrm{RM}(A)$. 
Theorem 7.2. Let $A$ be a $\sigma$-unital $C^{*}$-algebra whose dimensions of irreducible representations are bounded by an integer $n$. Then

$$
\mathrm{QM}(A)=\mathrm{LM}(A)+\mathrm{RM}(A) .
$$

Proof. We shall use induction on $n$.

Assume that Theorem 7.2 is true for all $n \leq k$. Let $n=k+1$ and $I=$ $\{x \in A: \pi(x)=0$, if $\operatorname{dim} \pi \leq k\}$. By [21, 4.4.10], $I$ is an ideal. Moreover, $I$ is a homogeneous $C^{*}$-algebra of order $n=k+1$. So $I$ arises from a locally trivial $M_{k+1}$-bundle [12]. Hence $\mathrm{QM}(I)=\mathrm{M}(I)$. Now $A / I$ is a $\sigma$-unital $C^{*}$ algebra whose irreducible representations have dimensions bounded by $k$. By the induction hypothesis, $\mathrm{QM}(A / I)=\mathrm{LM}(A / I)+\mathrm{RM}(A / I)$. It follows from Theorem 3.3 that $\mathrm{QM}(A)=\mathrm{LM}(A)+\mathrm{RM}(A)$.

Akemann and Shultz showed in [3] that a type I $C^{*}$-algebra is perfect if and only if every convergent sequence in $\widehat{A}$ converges to at most a countable number of points. So the algebras in Proposition 7.1 and Theorem 7.2 are perfect. We shall produce an imperfect $C^{*}$-algebra $A$, such that all of its irreducible representations are finite dimensional and $\mathrm{QM}(A)=\mathrm{LM}(A)+\mathrm{RM}(A)$.

Example 7.3. Let $H$ be a separable infinite dimensional Hilbert space and $\left\{H_{n}\right\}$ a sequence of mutually orthogonal, infinite dimensional subspaces. Let $e_{n}$ be the projection corresponding to $H_{n}$. There are sequences of finite rank projections $\left\{p_{i}^{n}\right\}$ together with a collection $\left\{q_{\sigma}^{n}\right\}$ of infinite rank projections indexed by binary strings $\sigma$ of 0 's and 1's such that

(i) $\sum p_{i}^{n}=e_{n}$ for each $n$,

(ii) $p_{i}^{n} q_{\sigma}^{n}=q_{\sigma}^{n} p_{i}^{n}$ for all $i, \sigma$ and $n$,

(iii) $q_{0}^{n}+q_{1}^{n}=e_{n}$ for each $n$,

(iv) $q_{\sigma_{0}}^{n}+q_{\sigma_{1}}^{n}=q_{\sigma}^{n}\left(e_{n}-p_{m}^{n}\right)$ for all $\sigma$, where $m=|\sigma|$ (see [3, Proposition 3.14]).

Let $I$ be the $C^{*}$-algebra of all compact operators on $H$ which commute with $\left\{p_{i}^{(n)}\right\}$. Let $A$ be the $C^{*}$-algebra generated by $I$ and by the set of projections $\left\{q_{\sigma}^{n}\right\}$.

We claim that $A$ is an imperfect, separable $C^{*}$-algebra all of whose irreducible representations are finite dimensional (and without identity). Clearly $I$ is an ideal of $A$. Moreover, $I$ is the restricted directed sum of finite dimensional ideals of $A$. Since the $q_{\sigma}^{n}$ 's commute with each other, $A / I$ is abelian. It follows that every irreducible representation of $A$ is finite dimensional. By [3, Proposition 3.14], $A$ is not perfect. By Theorem 3.3, $\mathrm{QM}(A)=\mathrm{LM}(A)+\mathrm{RM}(A)$.

\section{EXAMPLES}

Example 8.1. $\mathrm{QM}(A / I)=\mathrm{M}(A / I)$ and $\mathrm{QM}(I)=\mathrm{M}(I)$, but $\mathrm{QM}(A) \neq \mathrm{M}(A)$.

Let $A$ be the $C^{*}$-algebra of convergent sequences in $M_{2}$ with limits of the form $\left[\begin{array}{ll}* & 0 \\ 0 & 0\end{array}\right]$. Then it is easy to see that $\mathrm{QM}(A)$ consists of those bounded 
sequences $\left\{x_{n}\right\}_{n=1}^{\infty}$ in $M_{2}$ such that $\left(x_{n}\right)_{11} \rightarrow\left(x_{\infty}\right)_{11}$, whereas $\mathbf{M}(A)$ consists of those bounded sequences $\left\{x_{n}\right\}_{n=1}^{\infty}$ in $M_{2}$ such that $\left(x_{n}\right)_{11} \rightarrow\left(x_{\infty}\right)_{11}$, $\left(x_{n}\right)_{21} \rightarrow 0$ and $\left(x_{n}\right)_{12} \rightarrow 0$. Thus $\mathrm{QM}(A) \neq \mathrm{M}(A)$.

Let $I$ be the ideal of $A$ consisting of sequences $\left\{x_{n}\right\}_{n=1}^{\infty}$ in $M_{2}$ such that $x_{n} \rightarrow 0$. Then $\mathrm{QM}(I)=\mathrm{M}(I)$. Since $A / I$ is one dimensional, $\mathrm{QM}(A / I)=$ $\mathbf{M}(A / I)$.

Example 8.2. $\mathrm{QM}(A) \neq \mathrm{M}(A), \mathrm{QM}(A)=\mathrm{LM}(A)+\mathrm{RM}(A)$ but $\mathrm{QM}(I) \neq$ $\mathrm{LM}(I)+\mathrm{RM}(I)$.

Let $x$ be a countable compact Huasdorff space with $\lambda(x)=\omega$, where $\omega$ is the first limit ordinal. Let $\left\{e_{i j}\right\}$ be a set of matrix units for $K$.

Suppose $B_{0}=C(x) \otimes \dot{K}, B=\widetilde{B}_{0}$. Let $A$ be the $C^{*}$-algebra of convergent sequences in $B$ with limits in $C(x) \otimes e_{11}$. We identify $x \in B_{0}$ with an infinite matrix $\left(a_{i j}\right)$, where $a_{i j} \in C(x)$ is defined by $\left(1 \otimes e_{i i}\right) x\left(1 \otimes e_{j j}\right)=a_{i j} \otimes e_{i j}$. Identifying the identity of $\widetilde{B}_{0}$ with the identity matrix, we can identify elements of $\widetilde{B}_{0}$ with some infinite matrices. It is easy to check (by Theorem 1.4, for example) that $\mathrm{QM}(A)$ consists of these bounded sequences $\left\{\left(a_{i j}^{(n)}\right)\right\}_{n=1}^{\infty}$ in $B$ such that $a_{11}^{(n)} \rightarrow a_{11}^{\infty}$ and $\mathrm{M}(A)$ consists of those bounded sequences $\left\{\left(a_{i j}^{(n)}\right)\right\}_{n=1}^{\infty}$ in $B$ such that $a_{11}^{(n)} \rightarrow a_{11}^{\infty}$ and $a_{i j}^{(n)} \rightarrow 0$, if $i \cdot j \neq 1$, clearly $\mathrm{QM}(A) \neq \mathrm{M}(A)$. It follows from Lemma 4.6 that $\mathrm{QM}(A)=\mathrm{LM}(A)+\mathrm{RM}(A)$, since $B$ has an identity and $C(x) \otimes e_{11}$ is abelian. Let

$$
I=\left\{\left\{\left(a_{i j}^{(n)}\right)\right\}_{n=1}^{\infty}:\left(a_{i j}^{(n)}\right)=0 \text {, if } n \neq 1,\left(a_{i j}^{(n)}\right) \in B_{0}\right\} .
$$

Clearly $I$ is an ideal of $A$. It follows from Theorem 6.3 that $\mathrm{QM}(I) \neq \mathrm{LM}(I)+$ $\mathrm{RM}(I)$, since $I \cong C(x) \otimes K$.

Example 8.3. There is a separable antiliminal $C^{*}$-algebra $A$ such that $\mathrm{QM}(A)$ $\neq \mathrm{M}(A)$, but $\mathrm{QM}(A)=\mathrm{LM}(A)+\mathrm{RM}(A)$.

Let $B$ be the nonelementary separable matroid $C^{*}$-algebra with identity obtained as the inductive limit of the following

$$
M_{m(1)} \stackrel{g_{1}}{\longrightarrow} M_{m(2)} \stackrel{g_{2}}{\longrightarrow} M_{m(3)} \stackrel{g_{3}}{\longrightarrow} \cdots
$$

where $g_{i}(x)=x \otimes p$ and $\operatorname{dim} p=m(2) / m(1)$ (see [10]). Let $A_{0}$ be the $C^{*}$ subalgebra of $B$ generated by the elements $a$ such that $a \in M_{m(k)}$ for some $k, a=\left(a_{i j}\right), a_{i j}=0$, if $i j \neq 1$. Let $A$ be the $C^{*}$-algebra of convergent sequences $\{a(n)\}$ in $B$ with limits in $A_{0}$.

(1) $A$ is an antiliminal $C^{*}$-algebra. Let $I$ be a nontrivial ideal of $A$ and $I(k)=\{a(k): a \in I\}$. There is a smallest integer $k_{0}$ such that $I\left(k_{0}\right) \neq\{0\}$. Clearly, $I\left(k_{0}\right)$ is an ideal of $B$. Since $B$ is simple (see [10]), $I\left(k_{0}\right)=B$. Suppose $I_{0}=\left\{a \in I: a\left(k_{0}\right)=0\right\}$. Then $I_{0}$ is an ideal of $I$. Moreover $I / I_{0} \cong I\left(k_{0}\right)=B$. Thus $I$ is not liminal. So $A$ is an antiliminal $C^{*}$-algebra. 
(2) $\mathrm{QM}(A) \neq \mathrm{M}(A)$. Let $x$ be the sequence such that $x(n) \in M_{m(k)}$ for some $k$ and each $n$, moreover $(x(n))_{i j}=1$ for all $i, j \leq m(k)$, and $x(\infty)=$ $\left(a_{i j}^{\infty}\right)$, where $a_{11}^{\infty}=1, a_{i j}^{\infty}=0, i j \neq 1$. As in Example 8.1 and Example 8.2, one can easily check that $x \in \mathrm{QM}(A)$, but $x \notin \mathrm{M}(A)$.

(3) $\mathrm{QM}(A)=\mathbf{L M}(A)+\mathrm{RM}(A)$. Since $B$ has an identity, $\mathrm{M}(B)=\mathrm{QM}(B)=$ $B$. Moreover $A_{0}$ is abelian, so $\mathrm{M}\left(A_{0}\right)=\mathrm{QM}\left(A_{0}\right)$. It follows from Lemma 4.6 that $\mathrm{QM}(A)=\mathrm{LM}(A)+\mathrm{RM}(A)$.

\section{The DENSITY OF $\operatorname{LM}(A)+\operatorname{RM}(A)$ IN $\mathrm{QM}(A)$}

We know that $\mathrm{QM}(A) \neq \mathrm{LM}(A)+\mathrm{RM}(A)$, in general. But is $\mathrm{LM}(A)+\mathrm{RM}(A)$ dense in $\mathrm{QM}(A)$ in a suitable topology? (See [6, 7.2].)

Example 9.1. $\operatorname{LM}(A)+\mathrm{RM}(A)$ may not be norm closed.

Let $X$ be the one-point compactification of the disjoint union of $Y_{n}, n=$ $1,2, \ldots$ Let $A=C(X, K)$. Use the same notations in the proof of Theorem 6.3. Define

$$
F(t)=F_{n}(t) / \alpha\left(A_{n}\right)^{1 / 2}, \quad \text { if } t \in Y_{n}, \quad F(\infty)=0 .
$$

As in the proof of Lemma 4.7, we see that $F \in \mathrm{QM}(A)$, but $F \notin \mathrm{LM}(A)+$ $\operatorname{RM}(A)$. Let $G_{m}(t)=F(t)$, if $t \in Y_{n}, n \leq m, G_{m}(t)=0$, if $t \in Y_{n}, n>m$. Clearly $G_{m} \in \operatorname{LM}(A)+\operatorname{RM}(A)$ and $\left\|G_{m}(t)-F(t)\right\| \leq 1 / \alpha\left(A_{m}\right)^{1 / 2} \rightarrow 0$, as $m \rightarrow \infty$. Hence $\operatorname{LM}(A)+\operatorname{RM}(A)$ is not norm closed.

Proposition 9.2. Let $X$ be the disjoint union of $Y_{n}, n=1,2, \ldots$, and take $A=C_{0}(X, K)$. Then $\mathrm{LM}(A)+\mathrm{RM}(A)$ is not norm dense in $\mathrm{QM}(A)$.

Proof. Let $A_{n}=C\left(Y_{n}, K\right) \cong C\left(Y_{n}\right) \otimes K$. Take $x^{(n)} \in \mathrm{QM}\left(A_{n}\right)$ such that $\left\|x^{(n)}\right\| \leq 1$ and $\alpha\left(x^{(n)}\right) \geq \alpha\left(C\left(Y_{n}, K\right)\right)-1 / n$. Define $x(t)=x^{(n)}(t)$ if $t \in Y_{n}$. Assume that $u=y+z$, such that $y \in \operatorname{LM}(A), z \in \operatorname{RM}(A)$ and

$$
\|x-u\|<1 / 16 \text {. }
$$

Suppose $u=u^{(n)}(t), t \in Y_{n}, y=y^{(n)}(t), t \in Y_{n}$ and $z=z^{(n)}(t), t \in Y_{n}$, $n=1,2, \ldots$. Choose an integer $N$ such that

$$
\alpha\left(A_{N}\right) \geq \max (16,16 a),
$$

where $a=\max (\|y\|,\|z\|)$. Suppose $x^{(N)}=y_{1}^{(N)}+z_{1}^{(N)}$ and $x^{(N)}-u^{(N)}=$ $y_{2}^{(N)}+z_{2}^{(N)}$ such that $y_{1}^{(N)}, y_{2}^{(N)} \in \operatorname{LM}(A), z_{1}^{(N)} \in \operatorname{RM}(A)$ and $\left\|y_{2}^{(N)}\right\| \leq$ $(1 / 16)\left(\alpha\left(A_{N}\right)+1 / 21\right)$.

Let $\left\{e_{n}\right\}$ be an approximate identity for $A$ satisfying $e_{m} e_{n}=e_{n} e_{m}=e_{n}$, if $m>n$. By the proof of Lemma 2.1 and Theorem 2.3, there exists $n_{1}<n_{2}<\cdots$ such that

$$
\left\|\sum_{k=1}^{\infty}\left(1-e_{n_{k+1}}\right) y\left(e_{n_{k}}-e_{n_{k-1}}\right)\right\|<\frac{1}{12},
$$




$$
\begin{gathered}
\left\|\sum_{k=1}^{\infty}\left(1-e_{n_{k+1}}\right) z^{*}\left(e_{n_{k}}-e_{n_{k-1}}\right)\right\|<\frac{1}{12}, \\
\left\|\left.\sum_{k=1}^{\infty}\left(1-e_{n_{k+1}}\right) y_{i}^{(N)}\left(e_{n_{k}}-e_{n_{k-1}}\right)\right|_{Y_{N}}\right\|<\frac{1}{12}, \\
\left\|\left.\sum_{k=1}^{\infty}\left(1-e_{n_{k+1}}\right)\left(z_{i}^{(N)}\right)^{*}\left(e_{n_{k}}-e_{n_{k-1}}\right)\right|_{Y_{N}}\right\|<\frac{1}{12},
\end{gathered}
$$

$i=1,2$, and

$$
\left.\sum_{k=1}^{\infty}\left(1-e_{n_{k}}\right) x^{(N)}\left(e_{n_{k}}-e_{n_{k-1}}\right)\right|_{Y_{N}} \in \operatorname{RM}\left(A_{N}\right)
$$

Thus

$$
\left\|\left.\sum_{k=1}^{\infty}\left(1-e_{n_{k}}\right) x^{(N)}\left(e_{n_{k}}-e_{n_{k-1}}\right)\right|_{Y_{N}}\right\| \geq \alpha\left(A_{N}\right)-1-\frac{1}{N} .
$$

By the proof of Lemma 4.3,

$$
\left\|\sum_{k=1}^{\infty}\left(1-e_{n_{k}}\right) u\left(e_{n_{k}}-e_{n_{k-1}}\right)\right\| \leq 7 a+4
$$

and

$$
\left\|\left.\sum_{k=1}^{\infty}\left(1-e_{n_{k}}\right)\left(x^{(N)}-u^{(N)}\right)\left(e_{n_{k}}-e_{n_{k-1}}\right)\right|_{Y_{N}}\right\| \leq \frac{1}{16}\left(7 \alpha\left(A_{N}\right)+6\right) \leq \frac{1}{2} \alpha\left(A_{N}\right) .
$$

But

A contradiction. Hence

$$
\frac{1}{2} \alpha\left(A_{N}\right)+7 a+4<\alpha\left(A_{N}\right)-1-\frac{1}{n} .
$$

$$
\|x-u\| \geq 1 / 16
$$

Theorem 9.3. Let $A$ be a $C^{*}$-algebra. Then $\operatorname{LM}(A)+\operatorname{RM}(A)$ is strictly dense in $\mathrm{QM}(A)$. Moreover, for every $x \in \mathrm{QM}(A)$, there is a net $\left\{x_{\lambda}\right\} \subset \operatorname{LM}(A)+\operatorname{RM}(A)$ such that $\left\|x_{\lambda}\right\| \leq 2\|x\|$ and $x_{\lambda} \rightarrow x$ strictly. If $A$ is $\sigma$-unital, $\left\{x_{\lambda}\right\}$ can be taken as a sequence.

Proof. Take $x \in \mathrm{QM}(A)$ with $\|x\| \leq 1$. Let $\left\{e_{\lambda}\right\}$ be an approximate identity for $A$. Define $x_{\lambda}=e_{\lambda} x\left(1-e_{\lambda}\right)+x e_{\lambda}$. Clearly $e_{\lambda} x\left(1-e_{\lambda}\right) \in \operatorname{LM}(A), x e_{\lambda} \in$ $\mathrm{RM}(A)$.

For every $\varepsilon>0$ and $a \in A$, there is $\lambda_{0}$ such that if $\lambda \geq \lambda_{0}$, then $\left\|a\left(1-e_{\lambda}\right)\right\|<\varepsilon / 2$ and $\left\|\left(1-e_{\lambda}\right) a\right\|<\varepsilon / 2$. Thus

$$
\begin{aligned}
\left\|a\left(x_{\lambda}-x\right)\right\| & =\left\|a e_{\lambda} x\left(1-e_{\lambda}\right)-a x\left(1-e_{\lambda}\right)\right\| \\
& \leq\left\|a e_{\lambda}-a\right\|\left\|x\left(1-e_{\lambda}\right)\right\|<\varepsilon / 2<\varepsilon,
\end{aligned}
$$

and

$$
\begin{aligned}
\left\|\left(x_{\lambda}-x\right) a\right\| & =\left\|e_{\lambda} x\left(1-e_{\lambda}\right) a+x e_{\lambda} a-x a\right\| \\
& \leq\left\|e_{\lambda} x\right\|\left\|\left(1-e_{\lambda}\right) a\right\|+\|x\|\left\|\left(e_{\lambda}-1\right) a\right\|<\varepsilon .
\end{aligned}
$$


Moreover $\left\|x_{\lambda}\right\| \leq 2$. If $A$ is $\sigma$-unital, $\left\{e_{\lambda}\right\}$ can be taken as a sequence, so $\left\{x_{\lambda}\right\}$ is a sequence.

Let $X$ be the disjoint union of $Y_{n}, n=1,2, \ldots$, and take $A=C_{0}(X) \otimes$ $K$. It follows from Theorem 6.3 that $\mathrm{QM}(A) \neq \mathrm{LM}(A)+\mathrm{RM}(A)$. However, for every $x \in \mathrm{QM}(A)$, if we define $x_{n}(t)=x(t)$ for $t \in Y_{m}$ and $m \leq n$, $x_{n}(t)=0$ for $t \in Y_{m}$ and $m>n$, then $x_{n} \in \operatorname{LM}(A)+\operatorname{RM}(A)$ (Lemma 4.7), and $\left\|\pi^{* *}\left(x_{n}\right)-\pi^{* *}(x)\right\| \rightarrow 0$ uniformly on every compact subset of $\hat{A}$, with $\left\|x_{n}\right\| \leq\|x\|$. This type of density is stronger than the strict density considered in Theorem 9.3. Indeed, if $a \in A$, then $C=\{\pi \in \widehat{A},\|\pi(a)\| \geq \varepsilon\}$ is a compact subset of $\hat{A}$. Thus there is $N$ such that

$$
\left\|\pi(a)\left[\pi^{* *}\left(x_{n}\right)-\pi^{* *}(x)\right]\right\|<\varepsilon, \quad \pi \in C,
$$

and

$$
\left\|\pi(a)\left[\pi^{* *}\left(x_{n}\right)-\pi^{* *}(x)\right]\right\|<\varepsilon \cdot 2\|x\|,
$$

if $\pi \in \widehat{A} \backslash C$. From these inequalities, we see that $x_{n} \rightarrow x$ strictly. The construction of $x_{n}$ depends largely on the fact that $\hat{A}$ is Hausdorff. If $X$ is a countable locally (quasi-) compact space with $\lambda(X) \leq \infty$, we say $X$ satisfies condition (C), if for every $t \in X \backslash X_{[\infty]}$ there is an open set $O_{t}$ such that $t \in O_{t}$ and $\bar{O}_{t} \cap X_{[k]}=\varnothing$ for some $k$. Clearly, if $X$ is Hausdorff, then $X$ satisfies condition (C). If each point in $X \backslash X_{[\infty]}$ has a clopen neighborhood, then $X$ also satisfies condition $(\mathrm{C})$.

Theorem 9.4. Let $A$ be a separable $C^{*}$-algebra with countable spectrum $\hat{A}$ and $\widehat{A}_{[\infty]}=\varnothing$. If $\hat{A}$ satisfies condition (C), then for every $x \in \mathrm{QM}(A)$, there is a sequence $\left\{y_{n}\right\} \subset \operatorname{LM}(A)+\operatorname{RM}(A)$ such that $\left\|y_{n}\right\| \leq 3\|x\|$ and $\pi^{* *}\left(y_{n}\right)=\pi^{* *}(x)$ eventually on every compact subset of $\hat{A}$.

Proof. Take $x \in \mathrm{QM}(A)$ with $\|x\| \leq 1$. Put $I_{n}=\{a \in A: \pi(a)=0, \forall \pi \in$ $\left.\widehat{A}_{[n]}\right\}, n=1,2, \ldots$. Let $\left\{e_{i}\right\}$ be an approximate identity for $A$ and $\left\{p_{m}^{n}\right\}_{m=1}^{\infty}$ be an approximate identity for $I_{n}$. Define

$$
x_{i j}=\left(e_{i}-e_{i-1}\right)^{1 / 2} x\left(e_{j}-e_{j-1}\right)^{1 / 2} .
$$

Thus $x_{i j} \in A$, and since the norm closure $\bigcup_{n} I_{n}$ is $A$, we can find $\left\{p_{i}\right\} \subset$ $\left\{p_{m}^{n}, m, n=1,2, \ldots\right\}$ satisfying:

$$
\left\|x_{i j}\left(1-p_{j}\right)\right\|<\frac{1}{2^{i+j}}, \quad i \leq j,
$$

and

$$
\left\|\left(1-p_{i}\right) x_{i j}\right\|<\frac{1}{2^{i+j}}, \quad j \leq i .
$$

Define $p=\sum_{i=1}^{\infty}\left(e_{i}-e_{i-1}\right)^{1 / 2} p_{i}\left(e_{i}-e_{i-1}\right)^{1 / 2}$. Clearly $p \in \mathbf{M}(A)$. By Lemma 2.1, we see that $(1-p) x p+x(1-p) \in \operatorname{LM}(A)+\operatorname{RM}(A)$ (as in the proof of Lemma 3.2). Without loss of generality, we may assume that $p_{i} \in I_{i}$. 
Let $\widehat{A}=\left\{\pi_{1}, \pi_{2}, \ldots\right\}$. Fix $n$, and let $O_{n}$ be an open set of $\widehat{A}$ such that $\pi_{1}, \pi_{2}, \ldots, \pi_{n} \in O_{n}$ and $\bar{O}_{n} \cap \widehat{A}_{[k]}=\varnothing$ for some $k$. This is possible since $\hat{A}$ satisfies condition (C). Moreover, we may assume that $O_{n} \subset O_{n+1}$.

Let $J_{n}=\left\{a \in A ; \pi(a)=0, \forall \pi \in \bar{O}_{n}\right\}$. Clearly, if $\phi_{n}$ is the canonical homomorphism from $A$ to $A / J_{n}$, then $\phi_{n}\left(I_{k}\right)=\phi(A)$. Let $q_{i}$ be an element in $I_{k}$ such that $\left\|q_{i}\right\| \leq 1$ and $\phi_{n}\left(q_{i}\right)=\phi_{n}\left(p_{i}\right)$. Thus $\pi\left(q_{i}\right)=\pi\left(p_{i}\right)$ if $\pi \in \bar{O}_{n}$. Define

$$
q^{(n)}=\sum_{i=1}^{\infty}\left(e_{i}-e_{i-1}\right)^{1 / 2} q_{i}\left(e_{i}-e_{i-1}\right)^{1 / 2} .
$$

Then $q^{(n)} \in \mathrm{M}\left(A, I_{k}\right)$. Put $z_{n}=q^{(n)} x q^{(n)}$. Then $z_{n} \in \mathrm{QM}\left(A, I_{k}\right)$. It follows from Lemma 6.1 that $\mathrm{QM}\left(I_{k}\right)=\mathrm{LM}\left(I_{k}\right)+\mathrm{RM}\left(I_{k}\right)$. By Lemma 3.2, $z_{n} \in \operatorname{LM}\left(A, I_{k}\right)+\operatorname{RM}\left(A, I_{k}\right) \subset \mathrm{LM}(A)+\mathrm{RM}(A)$. Define $y_{n}=(1-p) x p+$ $x(1-p)+z_{n}$. Clearly $y_{n} \in \mathbf{L M}(A)+\operatorname{RM}(A)$. Moreover, $\left\|y_{n}\right\| \leq 3\|x\|$.

Let $S$ be a compact subset of $\widehat{A}, S=\left\{\pi_{1}, \pi_{2}, \ldots\right\}$. We have $\bigcup_{n} O_{n} \supset S$. Thus there are $n_{1}, n_{2}, \ldots, n_{m}$ such that $\bigcup_{j=1}^{m} O_{n_{j}} \supset S$. Since $O_{n} \subset O_{n+1}$, there is an integer $N$, such that $O_{N} \supset S$. If $n \geq N, \pi^{* *}\left(z_{n}\right)=\pi^{* *}($ pxp $)$ for $\pi \in \bar{O}_{N}$. Thus $\left\|\pi^{* *}\left(y_{n}\right)-\pi^{* *}(x)\right\|=0$ if $\pi \in S$.

Theorem 9.5. Let $A$ be a separable $C^{*}$-algebra of type I. Suppose that there is an integer $N$ such that for every $\pi \in \widehat{A}$, the closure $\{\pi\}^{-}$of $\{\pi\}$ is countable and $\lambda\left(\{\pi\}^{-}\right) \leq N$. Then for every $x \in \mathrm{QM}(A)$, there is a bounded net $\left\{x_{\alpha}\right\} \subset$ $\mathrm{LM}(A)+\operatorname{RM}(A)$ such that for every $\pi \in \hat{A}$

$$
\lim \left\|\pi^{* *}\left(x_{\alpha}\right)-\pi^{* *}(x)\right\|=0
$$

and $x_{\alpha} \rightarrow x$ strictly.

Proof. Let $\Gamma$ be the family of finite subsets of $\hat{A}$. Fix $\alpha \in \Gamma$. Then $\alpha^{-}$is countable. Moreover, $\lambda\left(\alpha^{-}\right) \leq \max \left\{\lambda\left(\{\pi\}^{-}\right), \pi \in \alpha\right\} \leq N$.

We may assume that $\|x\| \leq 1$. Let $J_{\alpha}=\bigcap_{\pi \in \alpha} \operatorname{ker} \pi$. Then $\left(A / J_{\alpha}\right)^{\wedge}$ is countable and $\lambda\left[\left(A / J_{\alpha}\right)^{\wedge}\right] \leq N$. Let $\phi: A \rightarrow A / J_{\alpha}$ be the canonical homomorphism from $A$ to $A / J_{\alpha}$. It follows from the proof of Lemma 6.1 that there are $\bar{y}_{\alpha}^{\prime} \in \operatorname{LM}\left(A / J_{\alpha}\right), \bar{y}_{\alpha}^{\prime \prime} \in \operatorname{RM}\left(A / J_{\alpha}\right)$ such that $\phi(x)=\bar{y}_{\alpha}^{\prime}+\bar{y}_{\alpha}^{\prime \prime},\left\|\bar{y}_{\alpha}^{\prime}\right\| \leq 3^{N}$ and $\left\|\bar{y}_{\alpha}^{\prime \prime}\right\| \leq 3^{N}$. It follows from [6] that there are $y_{\alpha} \in \operatorname{LM}(A)+\operatorname{RM}(A)$ such that $\phi\left(y_{\alpha}\right)=\bar{y}_{\alpha}^{\prime}+\bar{y}_{\alpha}^{\prime \prime}=\phi(x)$ and $\left\|y_{\alpha}\right\| \leq 2 \cdot 3^{N}$. Let $z_{\alpha}=x-y_{\alpha}$, then $\left\|z_{\alpha}\right\| \leq 2 \cdot 3^{N}+1$. Suppose that $\left\{e_{n}\right\}$ is an approximate identity for $A$. Define

$$
u_{\alpha}=e_{|\alpha|} z_{\alpha}\left(1-e_{|\alpha|}\right)+z_{\alpha} e_{|\alpha|}
$$

and $x_{\alpha}=y_{\alpha}+u_{\alpha}$. Clearly $x_{\alpha} \in \operatorname{LM}(A)+\operatorname{RM}(A)$ and $\left\|x_{\alpha}\right\| \leq 4 \cdot 3^{N}+2$. It is easy to check that

$$
\left\|\pi^{* *}\left(x_{\alpha}\right)-\pi^{* *}(x)\right\| \rightarrow 0
$$

for every $\pi \in \hat{A}$. Moreover, since $x-x_{\alpha}=z_{\alpha}-u_{\alpha}$, by the proof of Theorem 9.3 , we have $x_{\alpha} \rightarrow x$ strictly. 
Corollary 9.6. Let $A$ be a separable liminal $C^{*}$-algebra. Then for every $x \in$ $\mathrm{QM}(A)$, there is a bounded net $\left\{x_{\lambda}\right\} \subset \mathrm{LM}(A)+\mathrm{RM}(A)$ such that for every $\pi \in \widehat{A}$

$$
\lim \left\|\pi^{* *}\left(x_{\lambda}\right)-\pi^{* *}(x)\right\|=0
$$

and $x_{\alpha} \rightarrow x$ strictly.

Proof. $\widehat{A}$ is a $T_{1}$ space.

Note. The problem $\mathrm{QM}(A)=\mathrm{LM}(A)+\mathrm{RM}(A)$ for simple $C^{*}$-algebras has been studied and the results will appear elsewhere.

Acknowledgments. The author wishes to express his sincere thanks to his thesis advisor Professor Lawrence G. Brown. The author would also like to thank Professor Gert K. Pedersen for a critical reading of an earlier version of the paper.

\section{REFERENCES}

1. C. A. Akemann and G. K. Pedersen, Complications of semicontinuity in $C^{*}$-algebra theory, Duke Math. J. 40 (1973), 785-795.

2. C. A. Akemann, G. K. Pedersen and J. Tomiyama, Multipliers of $C^{*}$-algebra, J. Funct. Anal. 13 (1973), 277-301.

3. C. A. Akemann and F. Shultz, Perfect $C^{*}$-algebras, Mem. Amer. Math. Soc., vol. 55, no. 326 (1985).

4. L. G. Brown, Extensions of AF algebras: The projection lifting problem, Proc. Sympos. Pure Math., vol. 38, Part I, Amer. Math. Soc., Providence, R.I., 1982.

5. __ Stable isomorphisms of hereditary subalgebras of $C^{*}$-algebras, Pacific J. Math. 71 (1977), 335-348.

6. __ Closed hereditary $C^{*}$-algebras and the structure of quasi-multipliers, preprints.

7. $\ldots$ Semicontinuity and multipliers of $C^{*}$-algebras, preprint.

8. R. Busby, Doubly centralizers and extensions of $C^{*}$-algebras, Trans. Amer. Math. Soc. 132 (1968), 79-99.

9. J. Dixmier, Les $C^{*}$-algèbres et leurs représentations, Gauthier-Villars, Paris, 1964.

10. __, On some $C^{*}$-algebras considered by Glimm, J. Funct. Anal. 1 (1967), 182-203.

11. E. G. Effros, Dimensions and $C^{*}$-algebras, CBMS Regional Conf. Ser. in Math., no. 46, Amer. Math. Soc., Providence, R.I., 1980.

12. J. M. G. Fell, The structure of algebras of operator fields, Acta Math. 106 (1961), 233-280.

13. H. E. Jensen, Scattered $C^{*}$-algebras, Math. Scand. 41 (1977), 308-314.

14. __ Scattered $C^{*}$-algebras. II, Math. Scand. 43 (1978), 308-310.

15. B. E. Johnson, An introduction to the theory of centralizers, Proc. London Math. Soc. 14 (1964), 299-320.

16. $\ldots$, A counterexample in the perturbation theory of $C^{*}$-algebras, Canad. Math. Bull. 25 (1982), 311-316.

17. S. Mazurkiewicz and W. Sierpinski, Contribution à la topologie des ensembles dénombrables, Fund. Math. 1 (1920), 21.

18. A. J. Lazar, On scattered $C^{*}$-algebras, preprint.

19. H. Lin, The structure of quasi-multipliers of $C^{*}$-algebras, Ph.D. thesis, Purdue Univ., 1986.

20. K. McKennon, The quasi-multiplier conjecture, Proc. Amer. Math. Soc. 72 (1978), 258-260. 
21. G. K. Pedersen, Applications of weak ${ }^{*}$ semi-continuity in $C^{*}$-algebra theory, Duke Math. J. 39 (1973), 437-450.

22. __, C $C^{*}$-algebras and their automorphism groups, Academic Press, London, 1979.

23 _ $S A W^{*}$-algebras and corona $C^{*}$-algebras, contributions to non-commutative topology, $\mathrm{J}$. Operator Theory 15 (1986), 15-32.

Department of Mathematics, University of California, Santa Barbara, California 93106

Current address: Department of Mathematics, East China Normal University, 3663 Zhongshan Road (N), Shanghai 200062, China 